\title{
NONLINEAR VIBRATION ABSORBERS FOR ROPEWAY ROLLER BATTERIES CONTROL
}

\author{
Biagio Carboni ${ }^{1}$, Andrea Arena ${ }^{1}$ and Walter Lacarbonara ${ }^{1}$
}

\begin{abstract}
This work investigates a nonlinear passive control strategy designed to reduce the peak accelerations in ropeway roller batteries systems by deploying an array of nonlinearly visco-elastic vibration absorbers. The control effectiveness is compared with that of an equivalent array made of linearly visco-elastic absorbers. A nonlinear parametric model describing the interactions between the different parts of this mechanical multibody system previously developed by the present authors is here extended to include the passive vibration control system aimed to mitigate the acceleration peaks induced by the vehicles transit at different operational speeds. To this aim, a set of linearly visco-elastic vibration absorbers is first optimized through the Differential Evolution (DE) algorithm seeking to minimize the area below the frequency-response curves of the linear equations of motion. Then, a new group of nonlinearly visco-elastic absorbers, that can be largely tuned (i.e., they can exhibit either softening or hardening behaviors), is proposed to mitigate the accelerations induced in the roller by the vehicle transit. These nonlinearly visco-elastic absorbers are optimized by means of the DE algorithm and comparisons with the control achieved by the linear absorbers are carried out to show the higher performance of the proposed nonlinear device. A possible design of the nonlinearly visco-elastic absorber, based on the hysteresis of a wire rope assembly undergoing flexural cycles, is also proposed and discussed.
\end{abstract}

\section{Keywords}

Hysteretic absorbers, ropeways, vibration control, roller battery, nonlinear TMD

\section{Introduction}

Ropeways are cable-based transportation systems, adopted both in urban environment and mountain regions or sightseeing areas [1]. These systems are becoming a valid alternative to classical public transportation solutions. Yet to become more competitive, they require new design criteria so as to increase the number of passengers and their operational speeds. Such cableways are typically classified according to different geometric and mechanical characteristics such as the number of cables, the actuation system, the vehicle size and the mechanism to get in and out from the station $[2,3]$. Among them, mono-cable ropeways are an effective solution to cover long distances when small cabin sizes are employed. The studies present in the literature are mainly focused on the dynamic response of the vehicles to cross-wind loading and other dynamic effects $[4,5]$. A few works analyze the coupling between the cable motion and the swaying motion of the traveling vehicles [6]. Moreover, the investigation of the dynamical behavior is restricted to the evaluation of the roller battery natural frequencies and the interaction between rollers, cable, tower and vehicle is not taken into account [7]. Analytical approaches via parametric modeling were successfully adopted for modeling and predicting the response of cable-driven systems such as container cranes, and for designing passive vibration control devices $[8,9,10]$ to mitigate their oscillations.

By taking into account the nonlinear parametric model of ropeways compression roller batteries which was theoretically proposed and experimentally validated by the authors in [11, 12], a number of vibration absorbers is deployed on the different stages of the roller battery system to mitigate the accelerations due to the vehicle transit. The three stages of the roller battery system are depicted in Fig. 1 where the roller battery suspension point $C$ on the towersupported hoisting beam is the center of rotation for stage 1 , $B_{1}$ and $B_{2}$ are the centers of rotation for the second stage, $\left(A_{1}, A_{2}, A_{3}, A_{4}\right)$ are the centers of rotation for the third stage made of four pairs of sheaves.

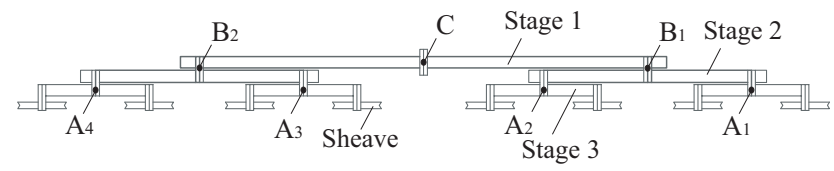

Figure 1. Top view of the roller battery with the three hierarchical stages and the sheaves.

Linear and nonlinear visco-elastic absorbers (VAs), are investigated and compared in this work. Moreover, the effectiveness of a newly conceived nonlinear control device is studied. The proposed model introduces a modulation of the stiffness and damping across the force-displacements cycles which can exhibit an asymptotically limited softening or hardening behavior. This is obtained by means of

\footnotetext{
${ }^{1}$ Department of Structural and Geotechnical Engineering Sapienza University of Rome

Rome, 00184, ITALY

Corresponding author:

Biagio Carboni

Email: biagio.carboni@uniroma1.it
} 


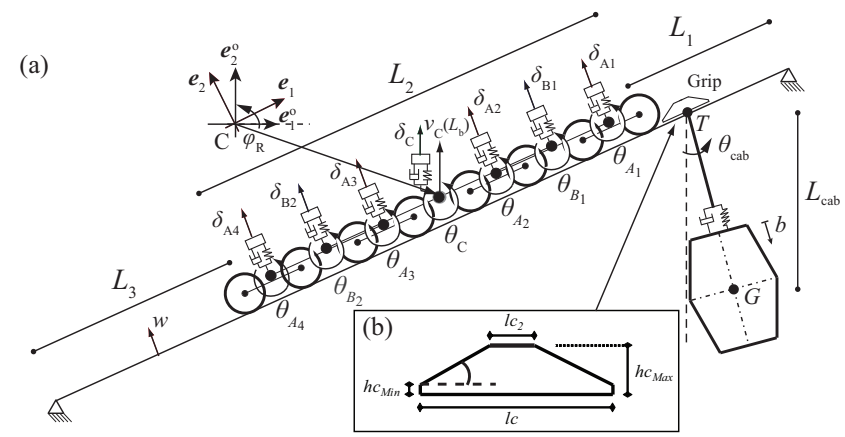

Figure 2. (a) Schematic representation of the cable-vehicle-roller battery assembly including the vibration absorbers arrangement. (b) Geometric characteristics of the grip.

displacement-dependent exponential functions adopted to model both the elastic stiffness and the viscous damping. Given the nonstationary nature of the dynamic loads induced by the transit of vehicles, instead of using the well-known formulas for the optimal linear TMDs tailored for purely harmonic excitations, the parameters of the visco-elastic absorbers are here evaluated performing an optimization based on the Differential Evolution (DE) algorithm [13, $14,15,16,17]$ which makes use of the frequency-response curves (FRCs) of the linearized equations of motion. The target of the optimization consists in the mitigation of the accelerations induced by skew-symmetric and nonsymmetric point-force distributions, respectively, acting on the roller battery sheaves. The optimal parameters obtained by making use of the linear FRCs are employed to identify the ranges in the optimization space of the mechanical parameters ruling the nonlinear visco-elastic absorbers. A DE algorithm-based optimization is carried-out to evaluate the parameters of the nonlinear absorbers delivering the best accelerations control in the case of high speed vehicle transit. A comparison between the performance of the linear and the nonlinear absorbers is shown and discussed. A possible design of the nonlinearly visco-elastic device, based on the preliminary results obtained in [18], capable of reproducing the desired nonlinear behavior is also proposed.

\section{Dynamic model of the cable-vehicle-roller battery assembly with the VAs}

The mechanical system here investigated is based on the parametric nonlinear formulation first proposed in [12]. The model takes into account the multibody assembly consisting of four interacting mechanical subsystems, namely, the elastic cable traveling along the ropeway line, the vehicle attached to the cable, the roller battery, and the hoisting beam connected to the tower (see Fig. 2). Moreover, the cable-rollers configuration investigated in this work refers to the so-called compression roller battery architecture, where the propelling cable moves below the rollers which are thus compressed against the hoisting beam.

The mentioned four substructures, interacting dynamically with each other, differ substantially in their mechanical behavior. In particular, the cable is an elastic onedimensional (1D) continuum which carries transverse loads by means of tensile forces tangent to the cable configuration.
On the other hand, the roller battery is a mechanism composed by rigid bodies (i.e., the rollers) rigidly connected to each other by means of hierarchical balancers rotating around moving hinges, and globally, around the master hinge fixed to the tip of the hoisting beam (see Fig. 1). The hoisting system, which connects the roller battery to the top part of the tower, is modeled as an equivalent 1D Euler beam element undergoing bending deflections. Finally, the vehicle behaves like a pendulum whose support is fixed to the moving cable and the equivalent vehicle mass is connected to the supporting arm via linearly visco-elastic supports positioned atop the vehicle. The connection between the vehicle and the cable is provided by the grip (see Fig. 2(b)), a mechanical element that works as a wedge and allows the vehicle transit below the rollers. The grip thus represents the interface through which dynamical interactions between the vehicle, the cable, and the roller battery occur.

The proposed mechanical model allows the investigation of the incremental dynamic response of the roller battery due to the vehicle transit across it. Due to the symmetry of the problem and the mechanical constraints of the roller battery, the motion of the system is assumed to be restrained within the plane containing the vertical (gravity) direction and the longitudinal (moving) direction. By considering the schematic representation of the roller battery depicted in Fig. 2 , let $\left(\mathbf{e}_{1}^{\mathbf{o}}, \mathbf{e}_{2}^{\mathrm{o}}\right)$ be the fixed frame, having its origin in the hinge $C$ and having $\mathbf{e}_{2}^{0}$ collinear with the vertical direction and $\mathbf{e}_{1}^{\mathrm{o}}$ with the longitudinal direction. A further fixed frame $\left(\mathbf{e}_{1}, \mathbf{e}_{2}\right)$, centered in $C$, is oriented along the roller inclined direction and is thus rotated counterclockwise with respect to $\mathbf{e}_{1}^{\mathrm{o}}$ by the angle $\varphi_{\mathrm{R}}$.

\section{Kinematic description}

The rollers assembly is made of four pairs of rollers, here modeled as rigid bodies, which rotate about the hinges denoted by $A_{j}(j=1, \ldots, 4)$ (see Fig. 1), while each couple of two rollers rotates about the hinges denoted by $B_{k}(k=$ $1,2)$. Finally, the main hinge $C$ is connected to the hoisting system and allows the eight rollers to rotate about the tip of the cantilever beam. The latter is modeled as an equivalent Euler-Bernoulli cantilever beam whose length $L_{b}$, bending stiffness $E_{b} I_{b}$, and mass $\rho A_{b}$ are determined so as to reflect the static and dynamic flexural behavior of the actual elastic structure connecting the roller battery to the tower.

Although the cable is the moving structural element of the system (i.e., the vehicle is propelled by the moving cable itself), the same modeling approach experimentally validated in [12] is here adopted. Thus the vehicle is modeled as a visco-elastic pendulum traveling across a stationary cable segment modeled as a prestressed string. This assumption is justified by the relatively low transit speeds. The equivalent string length $L_{e q}=L_{1}+L_{2}+L_{3}$ (see Fig. 2) was determined via an ad hoc experimental identification procedure described in [12], while $L_{\text {cab }}$ indicates the arm of the vehicle equivalent pendulum. Finally, the passive vibration absorbers (VA) are modeled as lumped masses connected through a rheological device to the roller battery at $A_{j}, B_{k}$, and $C$, respectively. In particular, the examined vibration absorbers take into account visco-elastic devices and nonlinearly visco-elastic devices, so as to compare the relative effectiveness for this nonstandard application. In 
particular, the nonlinear visco-elastic behavior is modeled according to a new model here proposed.

The roller battery degrees of freedoms (DOFs) are the rotations $\theta_{A_{j}}(t), \theta_{B_{k}}(t)$, and $\theta_{C}(t)$, about hinges $A_{j}, B_{k}$, and $C$, respectively. Therefore, the current configuration of the roller battery can be described by the vectors $\mathbf{r}_{P_{i}}(t)$, $\mathbf{r}_{A_{j}}(t), \mathbf{r}_{B_{k}}(t)$, and $\mathbf{r}_{C}(t)$ which provide at time $t$ the positions of the cable contact points $P_{i}(i=1, \ldots, 8)$ and those of the hinges $A_{j}(j=1, \ldots, 4), B_{k}(k=1,2)$ and $C$, respectively. On the other hand, the vehicle DOFs are denoted by $b(t)$ and $\theta_{c a b}(t)$ which indicate the elongation of the suspension system positioned atop the vehicle and the rotation about the suspension point at the grip, respectively. The vehicle moves along the cable at speed $v_{T}$ and the position at time $t$ of its center of mass is given by the vector $\mathbf{r}_{G}(t)$ while the position of the grip is described by the position vector $\mathbf{r}_{T}(t)$.

To discretize the time- and space-dependence function $v(x, t)$ representing the vertical displacement of the hoisting cantilever beam centerline, the Galerkin method is adopted and a suitable number $N_{l}$ of trial functions is employed to describe the dynamics of the beam-like subsystem. In particular, the lowest $N_{l}$ mode shapes $\psi_{l}(x)$ of the cantilever beam are employed to spoace-discretize the beam deflection while the functions $\eta_{l}(t)\left(l=1, \ldots, N_{l}\right)$ are introduced as time-dependent generalized coordinates. Therefore, the beam deflection can be written as $v(x, t) \approx \sum_{l=1}^{N_{l}} \psi_{l}(x) \eta_{l}(t)$ and the vertical displacement of point $C$ turns out to be $v_{\mathrm{C}}(t)=v\left(L_{b}, t\right) \approx \sum_{l=1}^{N_{l}} \psi_{l}\left(L_{b}\right) \eta_{l}(t)$

On the other hand, due to the multiple contact points $P_{i}(i=1, \ldots, 8)$ between the cable and the roller battery, the cable discretization is carried out according to the finite element (FE) technique. In particular, $n_{e}=20$ finite elements are used to divide the cable length such that each contact point $P_{i}$ coincides with a finite element node while the cable support points are the boundary nodes. Firstorder Lagrangian polynomials are employed to discretize the cable transversal displacement and to describe the kinematic function for the eth element. The latter can be written as $w_{e}(\xi, t)=\mathbf{N}_{e}(\xi) \mathbf{T}_{e} \mathbf{X}(t)$, where the subscript $e$ indicates the element, $\xi$ is the local arclength, $\mathbf{N}_{e}(\xi)$ is the $1 \times 2$ vector collecting the shape functions for each element nodes, $\mathbf{X}(t)$ is the vector of the nodal degrees of freedom, having size $\left(n_{e}+1\right) \times 1$, and $\mathbf{T}_{e}$ is the $2 \times n_{e}$ extraction matrix. Finally, due to the cable fixed boundary conditions, it turns out that $X_{1}(t)=X_{n_{e}+1}(t)=0$. The total number of DOFs for the described system is equal to 34 .

The unilateral contacts at points $P_{i}(i=1, \ldots, 8)$ between the rollers and the cable are modeled by introducing fictitious springs collocated at each point $P_{i}$. These springs behave as internal elastic constraints whose stiffness $K_{i}$ is suitably tuned in order to simulate a quasi-rigid behavior. The grip interaction with the rollers, arising from the vehicle transit, is reproduced by imposing a history of relative displacements between each roller and the cable defined according to a smoothed trapezoidal-in-time function derived from the grip shape and to the transit speed $v_{T}$. In particular, the prescribed upward displacement $g_{u}(t)$ and the downward displacement $g_{d}(t)$ of each roller, due to the passage of the grip, read:

$$
\begin{aligned}
& g_{u}(t)=h c_{M i n}+t \tan \theta_{0}+\frac{4}{l c_{1}^{2}}\left[3\left(h c_{M a x}-h c_{M i n}\right) v_{T}\right. \\
& \left.-l c_{1} \tan \theta_{0}\right] v_{T} t^{2}-\frac{4}{l c_{1}^{3}}\left[4\left(h c_{M a x}-h c_{M i n}\right) v_{T}\right. \\
& \left.-l c_{1} \tan \theta_{0}\right] v_{T}^{2} t^{3}, \\
& g_{d}(t)=\frac{1}{v_{T}\left(l c-l c_{2}\right)^{3}} \\
& \left\{v _ { T } \left[h c_{M i n}\left(5 l c-l c_{2}-4 v_{T} t\right)\left(l c+l c_{2}-2 v_{T} t\right)^{2}\right.\right. \\
& \left.-4 h c_{M a x}\left(l c+3 l c_{2}-4 v_{T} t\right)\left(l c-v_{T} t\right)^{2}\right] \\
& \left.+\left(l c-l c_{2}\right)\left(l c+l c_{2}-2 v_{T} t\right)^{2}\left(l c-v_{T} t\right) \tan \theta_{0}\right\} .
\end{aligned}
$$

Finally, the rollers kinematics prescribed by the vehicle transit and the grip interaction are described by the following expression:

$$
\begin{aligned}
& h_{i}(t)=\left(H\left(t-\tau_{P_{i}}-\tau_{0}\right)-H\left(t-\tau_{P_{i}}-\tau_{1}\right)\right) g_{u}\left(t-\tau_{P_{i}}\right) \\
& \quad+\left(H\left(t-\tau_{P_{i}}-\tau_{1}\right)-H\left(t-\tau_{P_{i}}-\tau_{3}\right)\right) h c_{M a x} \\
& \quad+\left(H\left(t-\tau_{P_{i}}-\tau_{3}\right)-H\left(t-\tau_{P_{i}}-\tau_{f}\right)\right) g_{d}\left(t-\tau_{P_{i}}\right)
\end{aligned}
$$

where $H$ is the Heaviside function, $\tau_{0}=0, \tau_{1}=\tau_{0}+\frac{l c_{1}}{2 v_{T}}$, $\tau_{3}=\tau_{0}+\frac{1}{2 v_{T}}\left(l c+l c_{2}\right), \tau_{f}=\tau_{0}+\frac{l c}{v_{T}}$, and $\tau_{P_{i}}$ represent the transit times below the $i$ th roller $P_{i}$. The values of the geometric and mechanical parameters presented in Eqs. (1) and (2) can be found in [12].

The vibration absorbers are positioned at the hinges $A_{j}$, $B_{k}$ and $C$, respectively. Such positioning is justified by the need of controlling the second stage (absorbers in $A_{j}$ ) and the first stage (absorbers in $B_{k}$ ) and the suspension point $C$ (absorber in $C$ ). The third stage is not directly controllable. The current configuration of the oscillating absorber masses is described in terms of the displacements $\delta_{A_{j}}$ and $\delta_{B_{k}}$, along the directions $\mathbf{e}_{2}^{A_{j}}$ and $\mathbf{e}_{2}^{B_{k}}$ orthogonal to the roller inclined configuration, and $\delta_{C}$ along the vertical direction $\mathbf{e}_{2}^{\mathbf{o}}$, respectively. Hence, the position of the VA masses can be described, in the fixed frame $\left(\mathbf{e}_{1}, \mathbf{e}_{2}\right)$, by the following vectors:

$$
\begin{aligned}
& \mathbf{r}_{\delta_{A_{j}}}(t)=\mathbf{r}_{A_{j}}+\delta_{A_{j}} \mathbf{R}_{\theta_{B_{k}}} \cdot \mathbf{e}_{2}^{A_{j}} \\
& \mathbf{r}_{\delta_{B_{k}}}(t)=\mathbf{r}_{B_{k}}+\delta_{B_{k}} \mathbf{R}_{\theta_{C}} \cdot \mathbf{e}_{2}^{B_{k}}, \\
& \mathbf{r}_{\delta_{C}}(t)=\left(v_{\mathrm{C}}\left(L_{b}\right)+\delta_{C}\right) \mathbf{R}_{\varphi_{\mathrm{R}}} \cdot \mathbf{e}_{2}^{\mathrm{o}}
\end{aligned}
$$

where $\mathbf{R}_{\theta_{B_{k}}}$ and $\mathbf{R}_{\theta_{C}}$ are the orthogonal matrices describing the rotations with respect to the hinges $B_{k}$ and $C$, respectively, while $\mathbf{R}_{\varphi_{\mathrm{R}}}$ rotates $\mathbf{e}_{2}^{\mathrm{o}}$ into the fixed frame $\left(\mathbf{e}_{1}, \mathbf{e}_{2}\right)$. On the other hand, at time $t$ the current configuration of the roller battery is described by the vectors $\mathbf{r}_{P_{i}}(t), \mathbf{r}_{A_{j}}(t), \mathbf{r}_{B_{k}}(t)$, and $\mathbf{r}_{C}(t)$ providing the positions $P_{i}$ $(i=1, \ldots, 8), A_{j}(j=1, \ldots, 4), B_{k}(k=1, \ldots, 2)$ and $C$, respectively, in the fixed frame $\left(\mathbf{e}_{1}, \mathbf{e}_{2}\right)$.

\section{Equations of motion}

The equations of motion governing the nonlinear dynamics of the roller battery including the passive control system can be obtained starting from the computation of the Lagrangian 
of the mechanical system. To this end, the kinetic energy $T(t)$ and the total potential energy $V(t)$ are first calculated as

$$
\begin{gathered}
T(t)=T_{b}(t)+T_{c a b}(t)+T_{\mathrm{R}}(t) \\
+\sum_{n=1}^{N_{\delta}} T_{\delta_{n}}(t)+\sum_{e=1}^{n e} T_{c, e}(t)\left(H\left(t-\tau_{e}\right)-H\left(t-\tau_{e+1}\right)\right), \\
V(t)=V_{b}(t)+V_{c}(t)+V_{e l, c a b}(t)+V_{f i c t}(t) \\
+\sum_{n=1}^{N_{\delta}} V_{\delta_{n}}(t)-V_{g, c a b}(t),
\end{gathered}
$$

where $V_{g, c a b}(t)$ is the potential energy of the cabin while $V_{e l, c a b}(t)$ and $V_{\text {fict }}(t)$ are the elastic energies due to the cabin suspension system and the fictitious springs, respectively. Moreover, $V_{b}(t), V_{c}(t)$, and $V_{\delta_{n}}(t)$ are the potential energies associated with the beam, the cable, and the $n$th VA, respectively, while $N_{\delta}$ is the total number of VAs. Finally, $T_{b}(t), T_{c a b}(t), T_{\mathrm{R}}(t), T_{\delta_{n}}(t)$, and $T_{c, e}(t)$ are the kinetic energies of the beam, the cable, the rollers system, the $n$th VA and the cabin passing across the eth finite element, respectively. The full expressions of each term in Eq. (4) can be found together with detailed comments in [12].

Finally, to model the energy dissipation due to the presence of the vibration absorbers, the two cases of linearly visco-elastic absorbers and nonlinearly visco-elastic absorbers are considered. In particular, in the first case, the Rayleigh dissipation function is considered to account for the viscous damping forces, while for the nonlinearly visco-elastic devices, a new rheological model is adopted to describe the restoring and dissipative forces. Therefore, once the system Lagrangian, $L(t)=T(t)-V(t)$, is computed, the equations of motion can be written as

$$
\frac{d}{d t}\left(\frac{\partial L}{\partial \dot{q}_{m}}\right)-\frac{\partial L}{\partial q_{m}}+d_{m} \dot{q}_{m}=\sum_{n=1}^{N_{\delta}} P_{\delta_{n}}^{(m)}
$$

where $\frac{d}{d t}$ and the overdot indicate total differentiation with respect to time $t, q_{m}$ is the $m$ th component of the vector collecting the system degrees of freedom, namely, $\mathbf{q}=\left[\theta_{A_{1}}\right.$ $\theta_{A_{2}} \theta_{A_{3}} \theta_{A_{4}} \theta_{B_{1}} \theta_{B_{2}} \theta_{C} \theta_{c a b} \quad b \quad X_{2} \ldots X_{n_{e}} \eta_{1} \ldots \eta_{N_{l}}$ $\left.\delta_{A_{1}} \ldots \delta_{A_{4}} \delta_{B_{1}} \delta_{B_{2}} \delta_{C}\right]^{\top}$, and $d_{m}$ is the equivalent linear damping coefficient for each degree of freedom. In the case of linearly visco-elastic absorbers, the expression of the potential energy assumes the classical form $V_{\delta_{n}}(t)=\frac{1}{2} k_{n} q_{n}^{2}$ ( $n=1, \ldots, N_{\delta}$ ), while the nonlinear restoring force of the absorbers can be obtained from the variation of the potential energy defined as follows

$$
V_{\delta_{n}}(t)=\frac{1}{2} k_{n}\left(q_{n}^{2}+\xi_{k, n} x_{k, n}^{2} e^{-q_{n}^{2} / x_{k, n}^{2}}\right) .
$$

Moreover, in Eq. (5) $d_{m}$ is the equivalent linear damping coefficient for each roller battery degree of freedom, while $P_{\delta_{n}}^{(m)}$ is the $m$ th generalized component of the $n$th VA viscous force; it turns out that $d_{m}=0$ when $m=\left(9+n_{e}+N_{l}+1\right), \ldots, N_{\delta}$, and $P_{\delta_{n}}^{(m)}=0$ when $m=$ $1, \ldots,\left(9+n_{e}+N_{l}\right)$. On the other hand, the Lagrangian force $P_{\delta_{n}}^{(m)}$ has the classical Rayleigh-type expression, $P_{\delta_{n}}^{(m)}=-d_{n} \dot{q}_{n}\left(n=1, \ldots, N_{\delta}\right)$, in the case of linear
VA, while, for the nonlinearly visco-elastic absorbers, the viscous damping force, the following displacementdependent nonlinear relationship holds:

$$
P_{\delta_{n}}^{(m)}=-d_{n}\left(1-\xi_{d, n} e^{-q_{n}^{2} / x_{d, n}^{2}}\right) \dot{q}_{n}
$$

In Eq. (6) $k_{n}$ denotes the elastic stiffness of the VA, while in Eq. (7) $d_{n}$ is the equivalent linear damping coefficient of each VA.

The constitutive parameters $\xi_{k, n} \in(-1,1)$ and $\xi_{d, n} \in$ $(-1,1)$ regulate the nonlinear contributions to of the elastic stiffness and the viscous damping forces, respectively. Depending on the sign of $\xi_{k, n}$, it is possible to confer a softening or a hardening behavior to the VAs; on the other end, the sign of $\xi_{d, n}$ regulates the rate of energy dissipation of the VAs. In particular, a positive value of $\xi_{d, n}$ produces pinching in the hysteresis cycle, thus it reduces the rate of dissipation at low displacements (i.e., in the neighborhood of the origin of the force-displacement diagram). In the present work, it is assumed $x_{k, n}=x_{d, n}=x_{n}$. It is a matter of fact that, by properly tuning the parameters associated with the nonlinear constitutive behavior of the VAs, the here proposed rheological model allows to design the nonlinearity of the absorbers so as to optimize their control authority over the structure with its peculiar nonlinearities to which the VAs are mounted.

Figure 3 shows the restoring force of the proposed nonlinearly visco-elastic absorber in the case of softening behavior and assuming $k_{n}=1, d_{n}=0.01$ and $x_{n}=0.5$; the cycles are generated for an assigned sinusoidal displacement with amplitude equal to 1 and circular frequency equal to $2 \pi$. In Fig. 3 (top) $\xi_{k, n}=\xi_{d, n}=-0.9$ and the softening behavior is associated with a viscous damping coefficient decreasing along the loading branch and determining a larger rate of dissipation around the origin. In Fig. 3 (bottom), where $\xi_{k, n}=-0.9, \xi_{d, n}=0.9$, the softening behavior is associated with a viscous damping coefficient increasing along the loading branch and determining a pinching of the cycle around the origin. The hardening case is reported in Fig. 4 by considering $k_{n}=1, d_{n}=0.01$ and $x_{n}=0.5$. Figure 4 (top) is obtained for $\xi_{k, n}=0.9$ and $\xi_{d, n}=-0.9$ while in Fig. 4 (bottom) $\xi_{d, n}=0.9$.

The equations of motion are then nondimensionalized by adopting $\omega_{c}=\sqrt{E_{b} I_{b} / \rho A_{b} L_{b}^{4}}$ as characteristic frequency and the equivalent hoisting beam span $L_{b}$ as characteristic length, respectively. The nondimensional semi-lengths of the balancers are assumed equal to $0.505,0.252$ and 0.126 for the 1st, 2nd and 3nd stage, respectively. The distances (orthogonal to the balancers in the vertical plane) between the hinges $\left(C, B_{k}, A_{j}\right)$ and center of the sheaves, in the reference configuration, are $0,0.0077$ and 0.023 , respectively. Moreover, the spans $L_{1}, L_{2}$ and $L_{3}$ are 0.177 , 1.19 and 0.88 , respectively (see Fig. 2 (a)). The inclination angle is assumed to be $\varphi_{R}=-0.253 \mathrm{rad}$. The geometric parameters of the grip are $h_{C, \text { Min }}=0, h_{C, \text { Max }}=0.022$, $l_{C}=0.431$, and $l_{C_{2}}=0.077$ (see Fig. 2 (b)) while the cabin equivalent length $L_{c a b}=1.19$. The nondimensional lumped cabin mass is 1.05 and the masses of the roller stages are 0.055 (at the center of the first and last sheave), 0.0416 (for the other remaining 6 sheaves), 0.0424 (at $A_{j}$ ), 0.0585 (at $B_{k}$ ), 0.0152 (at $C$ ). The cable nondimensional mass per 

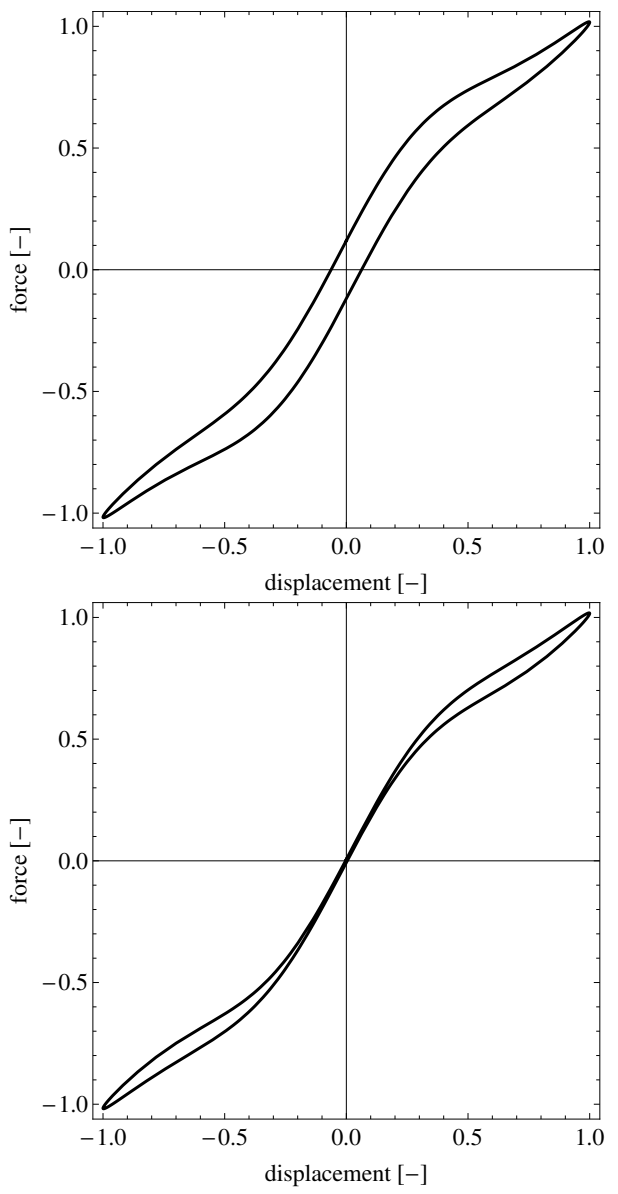

Figure 3. Nonlinearly visco-elastic force-displacement cycles in the softening case setting $k_{n}=1, d_{n}=0.01, x_{n}=0.5$ and $\xi_{k, n}=\xi_{d, n}=-0.9$ (top) and $\xi_{d, n}=0.9$ (bottom).

unit of length is 0.031 while the nondimensional tensile force in the reference configuration is equal to 0.009 . The nondimensional stiffness of the fictitious springs simulating the interaction between the cable and the rollers is set to 0.107 , while the nondimensional values of the stiffness and the equivalent damping ratio of the connection between cabin and suspension arm are equal to 0.074 and $7.0 \%$, respectively. Finally, the damping ratio for the pendular motion of the cabin is set to $8.0 \%$. The numerical simulations are carried out considering $N_{l}=4$ trial functions for the hoisting beam discretization, $n_{e}=20$ finite elements for the cable, while the total number of VAs is $N_{\delta}=7$. The mechanical and geometric properties of the roller battery assumed in this work are taken in consonance with the numerical and experimental results reported in $[11,12]$.

\section{Linear modal properties of the system}

To start with, a modal analysis is performed in order to evaluate the natural frequencies and the mode shapes of the system. Moreover, the modal masses are estimated according to the translational and rotational masses associated with each DOF of the system. To this end, the eigenvalue problem is studied neglecting the presence of the vehicle to understand the system modal sequence and frequencies spacing. It is also worth noting, that the geometric nonsymmetry of the system $\left(L_{1}<L_{3}\right.$, see Fig. 2) implies nonperfectly symmetric or skew-symmetric mode shapes.
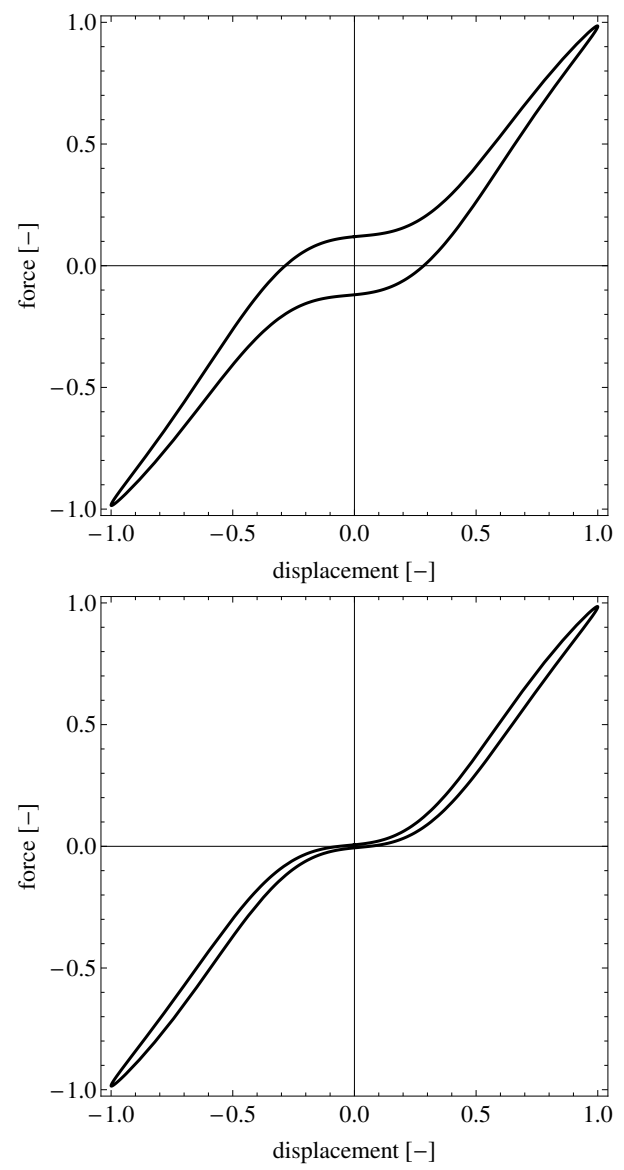

Figure 4. Nonlinearly visco-elastic force-displacement cycles in the hardening case setting $k_{n}=1, d_{n}=0.01, x_{n}=0.5$, $\xi_{k, n}=0.9$ and $\xi_{d, n}=-0.9$ (top) and $\xi_{d, n}=0.9$ (bottom).

Figures 5 and 6 show the modal coordinate contributions and the associated modal configurations of the lowest two modes of the system. To better understand the contribution of each generalized coordinate in the mode shapes of the roller battery, different colors are associated to each coordinate; in particular, red, orange and magenta refer to the rollers rotations $\theta_{A_{j}}, \theta_{B_{k}}$, and $\theta_{C}$, respectively, while blue and gray indicate the cable nodal DOFs listed in the vector $\mathbf{X}$ and the beam generalized coordinates $\eta_{l}$, respectively. As depicted in Fig. 5, the lowest mode shape is skew-symmetric, with a nondimensional frequency equal to 0.059 . The rollers rotate and are accompanied by nonnegligible rotations of the second and first stages; the latter denoted by $\theta_{C}$ (its presence characterizes all skew-symmetric modes) ensures that the hinge $\mathrm{C}$ is the point of skew-symmetry of the system mode shapes. On the other hand, the second mode shape shown in Fig. 6, having nondimensional frequency equal to 0.092 , is symmetric and shows the inner and the outer pairs of symmetric rollers undergoing opposite rotations of similar magnitude, while the second stages undergo equal and opposite rotations. Moreover, it is worth noting that the modal participation of the beam connected at $C$ to the roller battery becomes significant only for the eighth mode $(\bar{f}=0.279)$ characterized by a symmetric shape as shown in Fig. 7.

The damping ratios corresponding to each DOF of the system are: $\zeta_{A_{j}}=4.5 \%(j=1, \ldots, 4), \zeta_{B_{k}}=3.8 \%(k=$ $1,2), \zeta_{C}=3.8 \%$, while, for the supporting beam and 


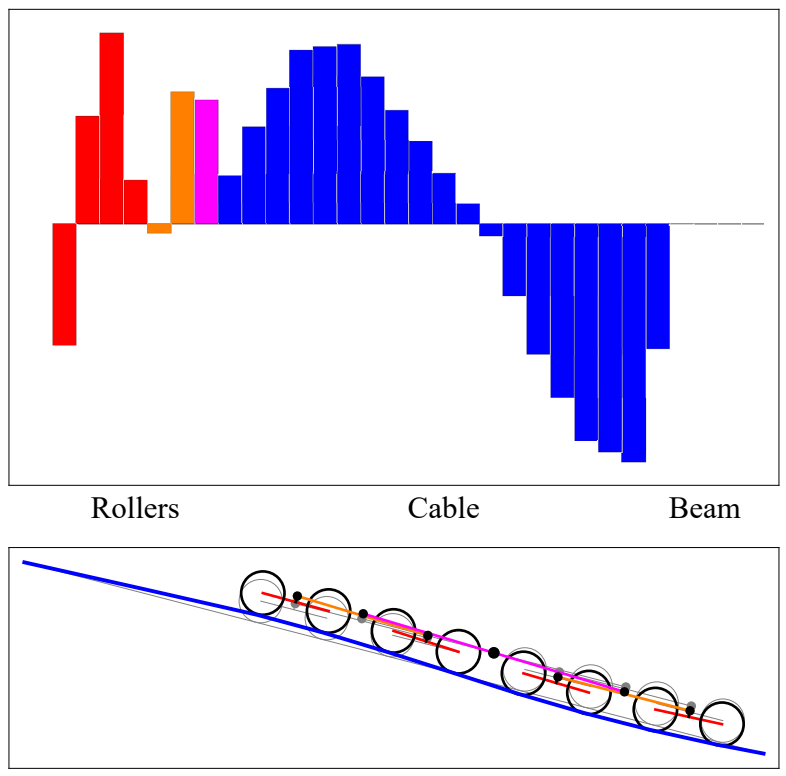

Figure 5. Lowest mode shape of the cable-tower-roller battery system.

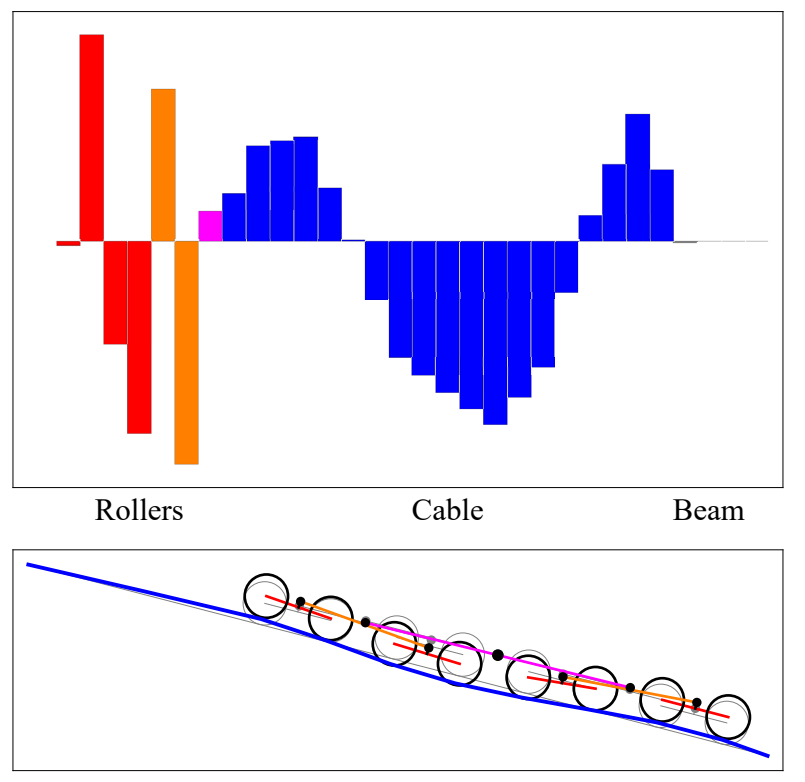

Figure 6. Second mode shape of the cable-tower-roller battery system.

the cable, the equivalent damping ratios are $2.2 \%$ and $1.5 \%$, respectively. The results of the eigenvalues analysis are shown in Tab. 1, where the nondimensional natural frequencies $\bar{f}$, the associated nondimensional masses $\bar{M}$, damping ratios $\zeta$, and stiffnesses $\bar{K}$ are reported for the lowest nine modes. The modal hierarchy of the system can be described as follows: the lowest modes are mainly associated with the rotation $\theta_{C}$ followed by the modes corresponding to the rotations $\theta_{B_{1}}, \theta_{B_{2}}$ and $\theta_{A_{2}}, \theta_{A_{3}}, \theta_{A_{1}}, \theta_{A_{4}}$. On the other hand, the eighth mode involves predominantly the dynamics of the hoisting beam.

\section{Vibration control of the roller battery}

Due to the size of the structural elements within the roller battery, feasible collocations of the VAs are found atop the hinges $A_{j}, B_{k}$, and $C$, respectively (see Fig. 2). Figure 8

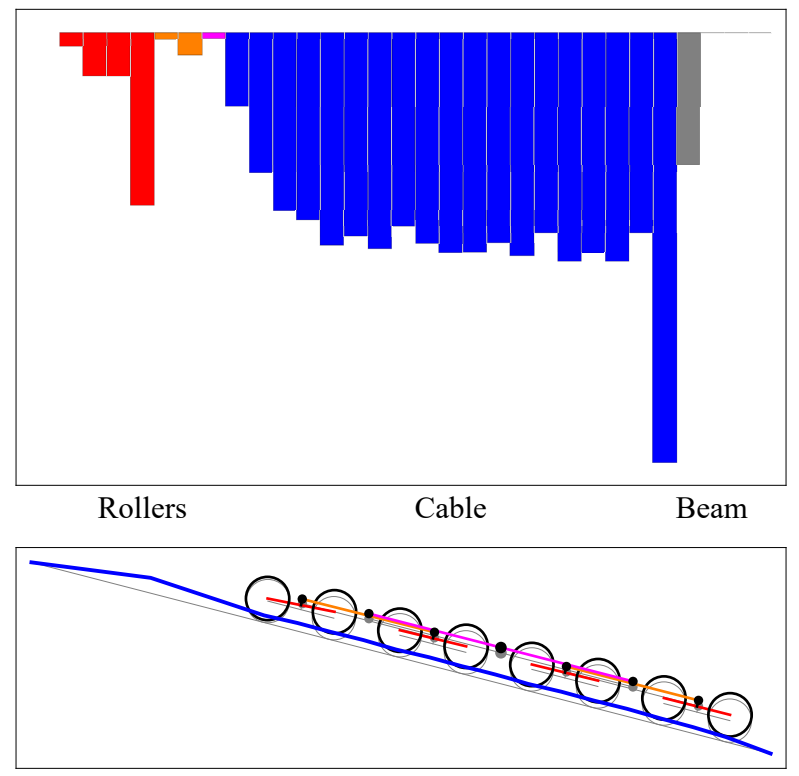

Figure 7. Eighth mode shape of the cable-tower-roller battery system.

Table 1. Lowest nine nondimensional natural frequencies, modal masses, damping ratios, and stiffnesses.

\begin{tabular}{c|cccc} 
Mode & $\bar{f}[-]$ & $\bar{M}[-]$ & $\zeta[\%]$ & $\bar{K}[-]$ \\
\hline 1 & 0.059 & $4.1 \cdot 10^{-2}$ & 1.03 & $5.8 \cdot 10^{-3}$ \\
2 & 0.092 & $2.7 \cdot 10^{-2}$ & 1.28 & $9.1 \cdot 10^{-3}$ \\
3 & 0.116 & $3.5 \cdot 10^{-2}$ & 1.05 & $1.9 \cdot 10^{-2}$ \\
4 & 0.152 & $9.2 \cdot 10^{-3}$ & 1.65 & $8.5 \cdot 10^{-3}$ \\
5 & 0.162 & $1.1 \cdot 10^{-2}$ & 1.51 & $1.1 \cdot 10^{-2}$ \\
6 & 0.176 & $9.8 \cdot 10^{-3}$ & 1.43 & $1.2 \cdot 10^{-2}$ \\
7 & 0.185 & $9.1 \cdot 10^{-3}$ & 1.38 & $1.2 \cdot 10^{-2}$ \\
8 & 0.279 & $4.2 \cdot 10^{-3}$ & 0.65 & $1.3 \cdot 10^{-2}$ \\
9 & 0.315 & $2.0 \cdot 10^{-3}$ & 0.46 & $8.3 \cdot 10^{-3}$ \\
\hline
\end{tabular}

shows the VAs configuration and highlights the rotational DOFs of the second stage and the vertical motion of the first stage to be controlled by the VAs (see also Fig. 1 for a top view of the roller battery). The devices in $A_{j}$ have control authority over the rotations $\theta_{B_{k}}$ and, in turn, on the rotation $\theta_{C}$ and vertical translation of $C$. The VAs collocated at $B_{k}$ work for both the rotation $\theta_{C}(t)$ and translation of $C$ while the absorber at $C$ has control authority mainly on the tip hoisting beam vertical motion and, in turn, on the vertical motion of the entire assembly. According to this hierarchy, the VAs positioned at $A_{1}, A_{2}, A_{3}$ and $A_{4}$ have the task of controlling the accelerations of these points whose motion is mainly due to the rotations $\theta_{B_{1}}$ and $\theta_{B_{2}}$, respectively. In turn, the absorbers at $B_{1}$ and $B_{2}$ are expected to mitigate the accelerations due to the rotation $\theta_{C}$. Thus, the VAs masses are defined considering the roller and cantilever total masses associated to each DOF. For the devices at $A_{j}$, the total mass is set to $1 \%$ of the total rotational mass associated to $\theta_{B_{1}}$ and $\theta_{B_{2}}$. It is equally distributed in the 4 VAs and the equivalence between rotational and translational mass is computed according to the rigid arms of the balancers forming the second stage. Specifically, the translational mass of each of the 4 absorbers at $A_{j}$ is assumed equal to $1 \%$ of a quarter of the rotational mass associated to $\theta_{B_{1}}$ and $\theta_{B_{2}}$ 
divided by the square of the distance between the point where the VA is positioned and the rotation center (i.e. the distance between $A_{1}$ or $A_{2}$ and $C_{1}$ and the distance between $A_{3}$ or $A_{4}$ and $C_{2}$ ). In the same way, the masses of the VAs at $B_{1}$ and $B_{2}$ are assumed to be $1 \%$ of the total mass associated to $\theta_{C}$. Finally, the mass of the absorber at $C$ is set to $1 \%$ of the translational mass related to the vertical motion of the beam tip. The VAs translational masses are reported in Tab. 2.

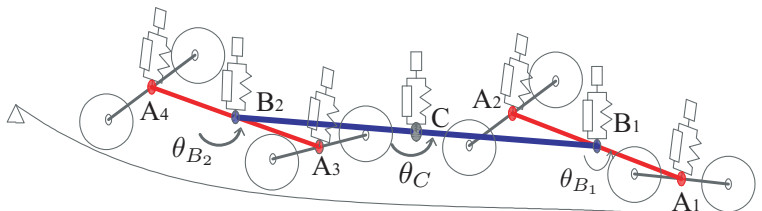

Figure 8. Configuration of the VAs and associated rotational DOFs to be controlled, namely the second stage rotations and the first stage vertical motion.

Table 2. VAs translational masses.

\begin{tabular}{cccc}
\hline $\mathrm{VA}_{A_{1}}$ & $\mathrm{VA}_{A_{2}}$ & $\mathrm{VA}_{A_{3}}$ & $\mathrm{VA}_{A_{4}}$ \\
\hline 0.00128 & 0.00128 & 0.00128 & 0.00128 \\
\hline \multicolumn{4}{c}{} \\
\hline $\mathrm{VA}_{B_{1}}$ & $\mathrm{VA}_{B_{2}}$ & $\mathrm{VA}_{C}$ \\
\hline 0.003193 & 0.003193 & 0.0166 \\
\hline
\end{tabular}

A preliminary concept design of the nonlinear absorber is shown in Fig. 9. The device hosted by a cylindrical enclosure features a mass that can oscillate vertically along a shaft. A group of steel wire ropes are connected at one end to the inner wall of the cylinder and at the other end to some plates connected with the oscillating cylindrical mass which moves along the vertical shaft fixed to the cylindrical enclosure ends. The connections between the wire ropes and the cylinder are such that the wire ropes are subject only to flexural stresses in order to provide the desired restoring force according to the design principles discussed in [18]. Another possibility consists in adopting a connection that induces tensile forces in the ropes when the absorber cylindrical mass slides on the shaft. In this case, the nonlinearly visco-elastic restoring force is characterized by a strong hardening that can be useful to mitigate the roller response subject to the impact of the grip. The cylindrical VAs are rigidly connected to the different stages of the roller battery.

\section{Optimization of the linear VAs}

The VA effectiveness in mitigating the accelerations of the roller battery components due to the vehicle transit across the tower is investigated next. The optimal parameters of the absorbers are estimated according to a simplified linear analysis based on the configuration which incorporates the visco-elastic VAs. Two harmonic loading conditions are considered, the first is given by a system of point-forces $F_{i}(t)=$ $F(1-2(8-i) / 7) \cos \Omega t(i=1, \ldots, 8)$. These forces are skew-symmetrically distributed across the roller battery and act at all points $P_{i}\left(F=4.14 \cdot 10^{-7}\right)$. The second loading condition given by a nonsymmetric distribution in which the
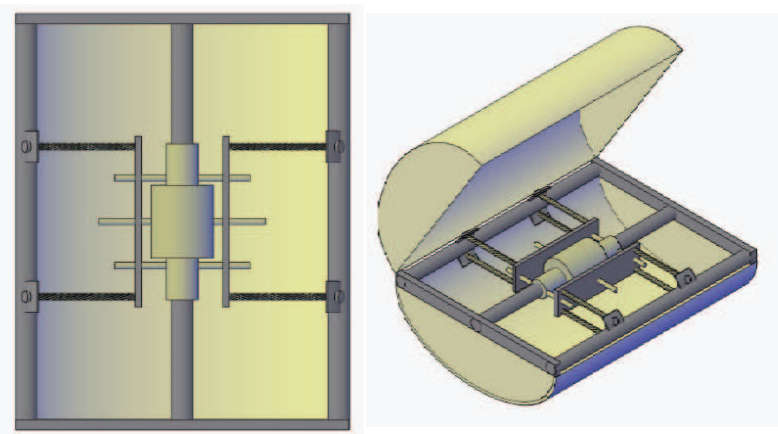

Figure 9. Preliminary concept design of the nonlinearly visco-elastic vibration absorber.

force acting at $P_{1}$ is zero (i.e., $F_{1}(t)=0$ ), while the force at point $P_{8}, F_{8}(t)=2 F \cos \Omega t$. The force acting at $P_{i}$ can be written as $F_{i}(t)=2 F(1-(8-i) / 7) \cos \Omega t(i=1, \ldots, 8)$.

The FRCs are analytically calculated by linearizing the system of equations about the reference configuration and computing the frequency response. The adopted VAs optimization criterion is based on the minimization of the objective function $\Lambda$ given by the area subtended by the FRCs in terms of accelerations of $P_{i}, A_{j}, B_{k}$, and $C$ :

$$
\begin{aligned}
\Lambda= & \int_{f_{0}}^{f_{1}} a_{C} d f+\sum_{k=1}^{2} \int_{f_{0}}^{f_{1}} a_{B_{k}} d f \\
& +\sum_{j=1}^{4} \int_{f_{0}}^{f_{1}} a_{A_{j}} d f+\sum_{i=1}^{8} \int_{f_{0}}^{f_{1}} a_{P_{i}} d f .
\end{aligned}
$$

The terms $f_{0}$ and $f_{1}$ are the lower and upper frequency bounds of the range over which the FRCs of the accelerations $a_{C}, a_{B_{k}}, a_{A_{j}}$ and $a_{P_{i}}$ are computed. The choice of minimizing the accelerations over the whole frequency bandwidth is prompted by the requirement of globally mitigating the vibrations in the multi-body system. The parameters to be optimized are represented by the resonance frequencies and the damping ratios of each absorber and the associated research spaces are defined according to a normal distribution around average values.

In accordance with the linear absorbers literature [19, 20], the optimal frequency and damping ratio are close to those of the structure to be controlled. For the present system, we can identify 2 search ranges for the frequency and 2 for the damping ratios. Considering the mechanical coupling between the rotational DOFs, the research space for the resonance frequencies of the VAs in $A_{j}$ and $B_{k}$ is taken to cover the range of frequencies associated with the lowest 7 modes (i.e., the rotational modes). On the other end, the frequency search space for the VA in $C$ is set around the frequency of the 8 th mode (i.e., the translational mode). The same criterion is employed for the damping ratios. The parameter population is constituted by 14 rows (number of parameters) and 25 columns (number of discrete values assumed in the search space) and the mutation coefficient of the DE algorithm is set to 0.9. The DE, at each iteration, generates a new parameter population that is improved according to the minimization of the $\Lambda$ (8). The iterations are stopped when a convergence of the $\Lambda$ is achieved. A detailed description of the DE algorithm steps can be found in $[21,13,14]$. 
Two sets of optimal parameters are found for the skewsymmetric and nonsymmetric loading conditions (see Tab. $3)$. The computed FRCs in accelerations of $P_{8}, A_{4}, B_{2}$, and $C$, are shown in Figs. 10 and 11, respectively, for the skew-symmetric loading scenario in the controlled and uncontrolled configurations, respectively. The same kind of results concerning $P_{4}, A_{2}, B_{1}$, and $C$ for the nonsymmetric load distribution are reported in Figs. 12 and 13, respectively. Figure 14 shows that the normalized $\Lambda$ for the skew-symmetric and the nonsymmetric loading condition converges monotonically to its minimum after only 350 iterations. As shown in Tab. 3, the natural frequencies of the absorbers optimized for the skew-symmetric loading condition span the range that covers the lowest five modes of the uncontrolled system (see Tab. 1) in the case of the VAs positioned at points $A_{j}$ and $B_{k}$, while the frequency of the VA positioned in $C$ lies between the frequency of the 8th and 9 th mode of the uncontrolled system. On the other hand, the natural frequencies of the absorbers optimized for the nonsymmetric loading condition span in the range that covers the lowest eight modes for all VAs. In particular, the natural frequency of the absorber at $B_{1}$ lies between the frequencies of the 6 th and 7 th mode of the uncontrolled system, while that of the absorber at $B_{2}$ varies between the frequencies of the 8 th and 9 th modes.

The frequency-response curves of the controlled system, reported in Figs. 10 and 11 for the case of skewsymmetric loading scenario, show resonance peaks around the frequency of the lowest uncontrolled mode. This is due to the large excitation induced by the skew-symmetric pointloads distribution in the lowest uncontrolled mode which drives the optimization of the absorbers at $A_{4}, B_{1}$ and $B_{2}$ around its natural frequency. On the other hand, the VAs optimization for the nonsymmetric point-loads (see Figs. 12 and 13) determines FRCs that show a double peak around almost each of the natural frequencies of the uncontrolled system.

The modal properties of the roller battery system including the presence of the VAs, calculated by solving the corresponding eigenvalues problem, are reported in Tabs. 4 and 5 , for the case of VAs constitutive parameters optimized for the skew-symmetric and the nonsymmetric loading scenarios, respectively. It is worth noting that the optimal damping ratios of the linear VAs positioned at $A_{i}$ $(i=1, \ldots, 8)$ assume almost the same value in both loading conditions, while the absorbers positioned at $B_{k}$ and $C$ are optimized with different damping values, and this is due to the fact that they are tuned to different frequencies.

\section{Optimization of the nonlinear VAs}

An effective strategy for performing the optimization of the nonlinear VAs consists in employing equivalence relationships for tuning of resonance frequency and damping with those of the optimal linear VAs obtained according to the linearized FRCs [22]. However, such approach does not ensure an overall optimal control performance considering the transient nature of the excitation. Therefore, the optimization of the nonlinearly visco-elastic absorbers is here achieved by performing numerical time integration of the nonlinear equations of motion including the transit of the cabin across the roller battery. The optimal parameters
Table 3. Nondimensional optimal resonance frequencies and damping ratios of the linear visco-elastic VAs for the skew-symmetric and nonsymmetric point-load distributions, respectively.

\begin{tabular}{ccccc}
\hline & \multicolumn{2}{c}{ Skew-symmetric } & \multicolumn{2}{c}{ Nonsymmetric } \\
VA & $\bar{f}_{\text {opt }}[-]$ & $\zeta_{\text {opt }}[\%]$ & $\bar{f}_{\text {opt }}[-]$ & $\zeta_{\text {opt }}[\%]$ \\
\hline$A_{1}$ & 0.158 & 0.69 & 0.158 & 0.68 \\
$A_{2}$ & 0.115 & 3.13 & 0.115 & 3.10 \\
$A_{3}$ & 0.095 & 3.55 & 0.095 & 3.78 \\
$A_{4}$ & 0.057 & 2.62 & 0.059 & 2.87 \\
$B_{1}$ & 0.063 & 2.56 & 0.178 & 0.78 \\
$B_{2}$ & 0.060 & 2.66 & 0.321 & 1.33 \\
$C$ & 0.295 & 5.50 & 0.281 & 6.38 \\
\hline
\end{tabular}
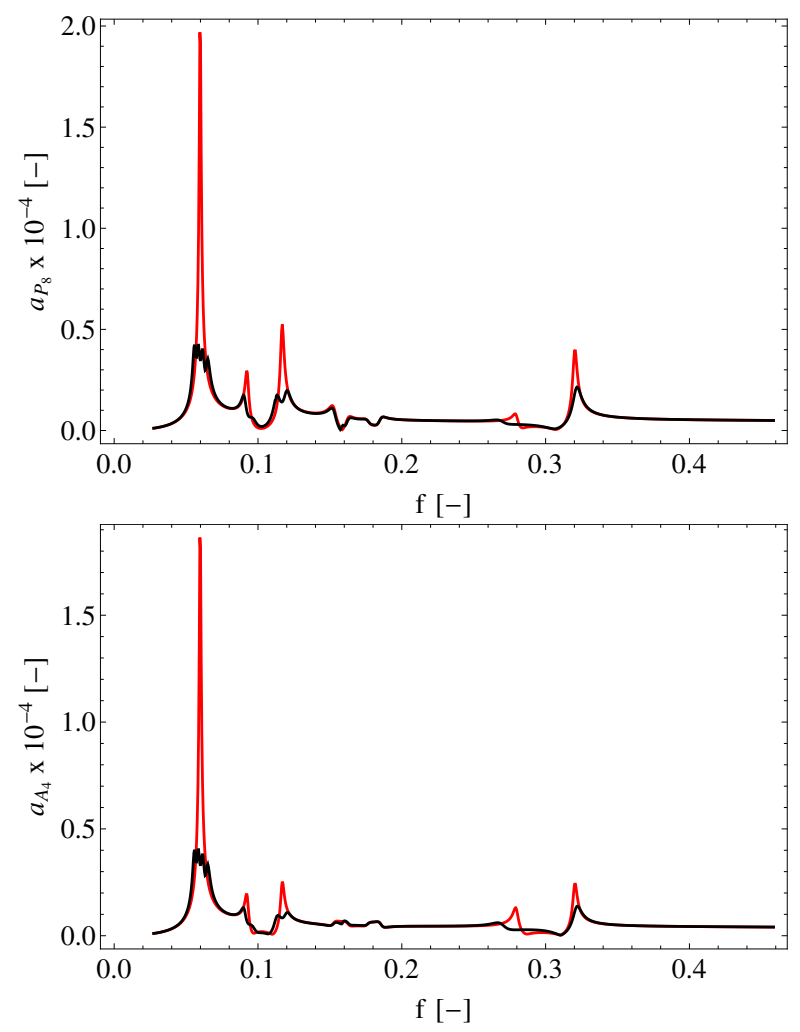

Figure 10. Controlled (black line) vs. uncontrolled (red line) FRCs of the roller battery points (top) $P_{8}$ and (bottom) $A_{4}$ for the skew-symmetric load distribution on the rollers.

of the nonlinear VAs are computed for the transit of the vehicle at the nondimensional speed of 0.025 using the DE algorithm. The selected value of the vehicle speed is quite larger with respect to the typical value of the transit speed for passengers transportation. Indeed, higher transit speeds may cause vibrations amplitude generating discomfort for passengers or it may cause overstress or fatigue problems in the ropeway structural elements. The possibility of mitigating the accelerations induced in the roller battery can be then a valid solution for increasing the running speeds while preserving the structural integrity. 

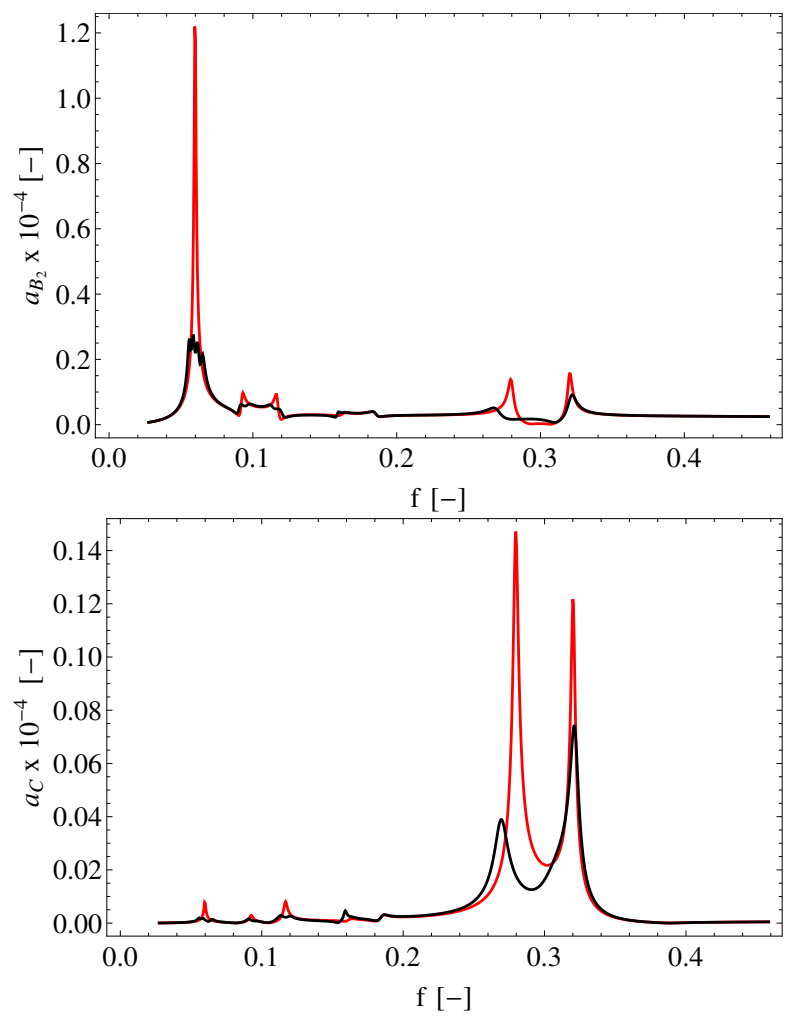

Figure 11. Controlled (black line) vs. uncontrolled (red line) FRCs of the roller battery points (top) $B_{2}$ and (bottom) $C$ for the skew-symmetric load distribution on the rollers.
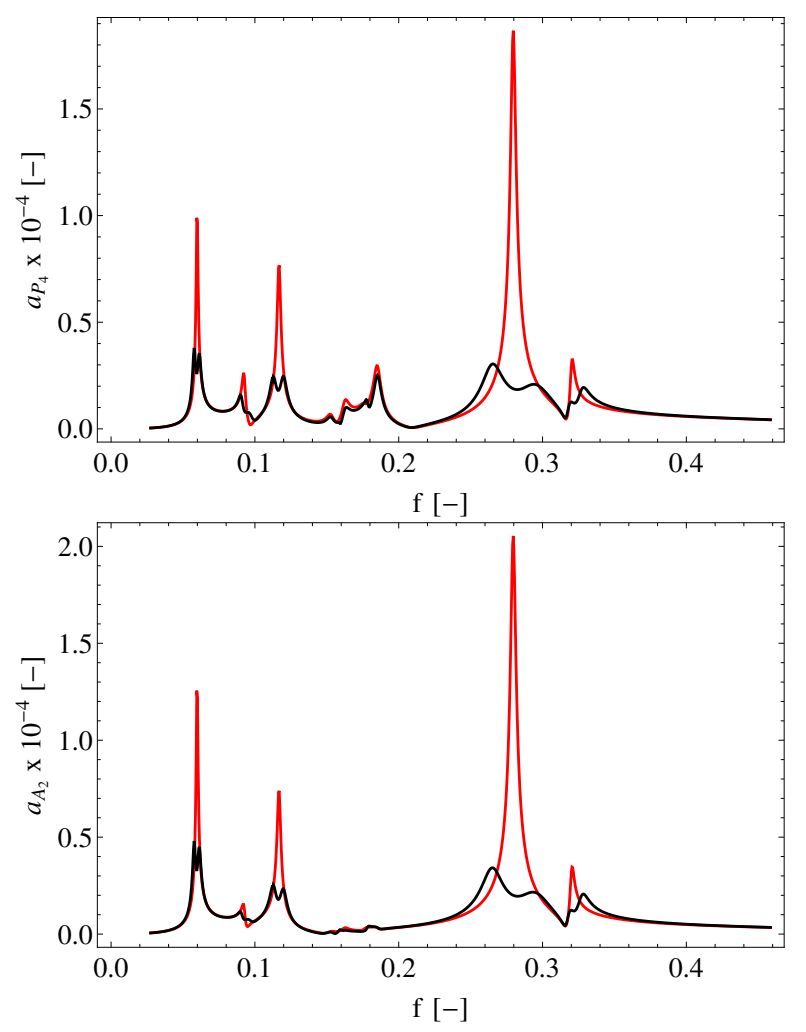

Figure 12. Controlled (black line) vs. uncontrolled (red line) FRCs of the roller battery points (top) $P_{4}$, (bottom) $A_{2}$ for the nonsymmetric load distribution on the rollers.

The network of the nonlinear VAs is optimized by the minimization of the following objective function:

$$
\Gamma=\int_{t_{0}}^{t_{f}}\left|a_{C}\right| d t+\sum_{k=1}^{2} \int_{t_{0}}^{t_{f}}\left|a_{B_{k}}\right| d t
$$

$\stackrel{\text { Prepared using sagej.cls }}{+} \sum_{j=1}^{4} \int_{t_{0}}^{t_{f}}\left|a_{A_{j}}\right| d t$
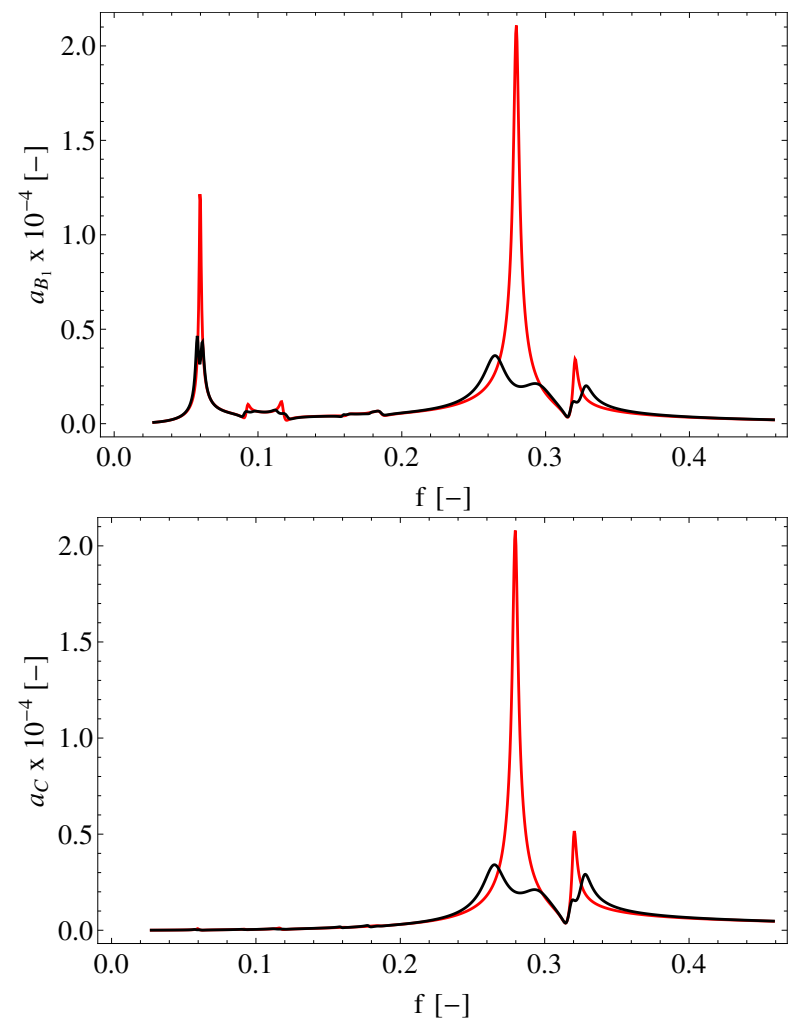

Figure 13. Controlled (black line) vs. uncontrolled (red line) FRCs of the roller battery points (top) $B_{1}$ and (bottom) $C$ for the nonsymmetric load distribution on the rollers.
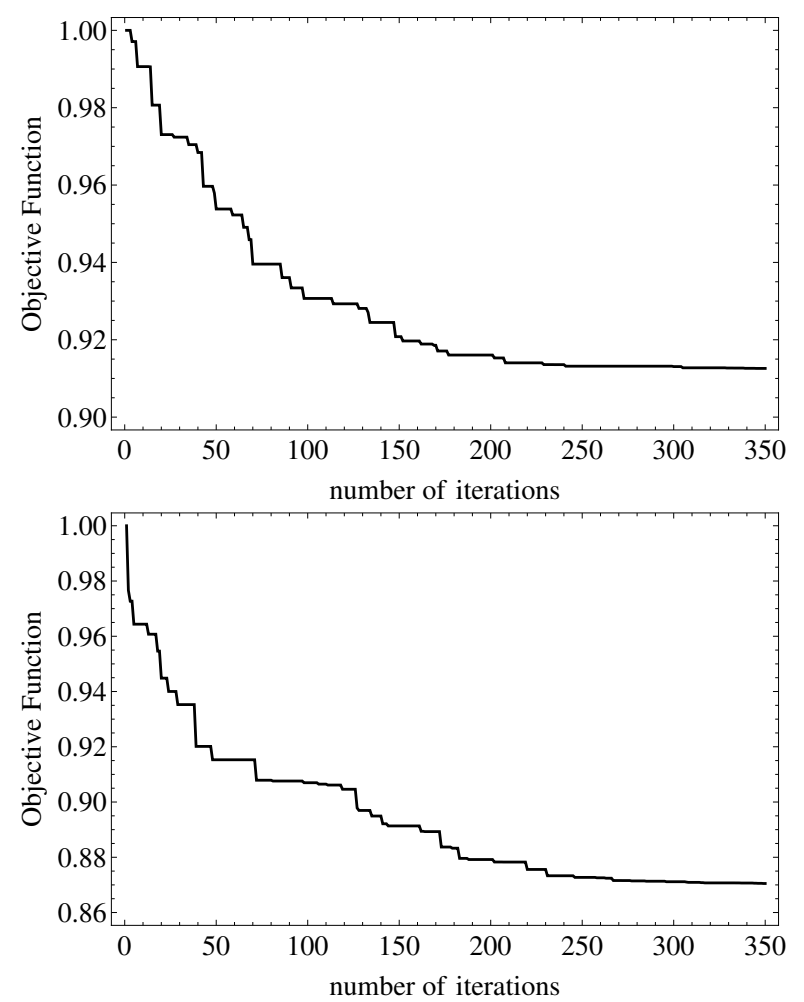

Figure 14. Normalized objective function vs. number of iterations performed by the DE algorithm for the skew-symmetric (top) and nonsymmetric (bottom) harmonic loading case.

where $t_{0}$ and $t_{1}$ represent the vehicle initial and final transit times across the equivalent cable length $L_{e q}$, respectively. Moreover, it is worth mentioning that only the accelerations 
Table 4. Lowest sixteen nondimensional natural frequencies, modal masses, damping ratios, and stiffnesses of the system including linear visco-elastic vibration absorbers optimized according to the skew-symmetric point-load distribution.

\begin{tabular}{c|cccc} 
Mode & $\bar{f}[-]$ & $\bar{M}[-]$ & $\zeta[\%]$ & $\bar{K}[-]$ \\
\hline 1 & 0.056 & $7.50 \cdot 10^{-4}$ & 2.07 & $9.28 \cdot 10^{-5}$ \\
2 & 0.058 & $9.60 \cdot 10^{-4}$ & 2.15 & $1.29 \cdot 10^{-4}$ \\
3 & 0.061 & $1.27 \cdot 10^{-3}$ & 2.26 & $1.89 \cdot 10^{-4}$ \\
4 & 0.064 & $1.03 \cdot 10^{-3}$ & 2.35 & $1.71 \cdot 10^{-4}$ \\
5 & 0.090 & $3.11 \cdot 10^{-3}$ & 1.87 & $1.00 \cdot 10^{-3}$ \\
6 & 0.097 & $6.5 \cdot 10^{-4}$ & 2.95 & $2.41 \cdot 10^{-4}$ \\
7 & 0.112 & $1.25 \cdot 10^{-3}$ & 2.12 & $6.26 \cdot 10^{-4}$ \\
8 & 0.120 & $1.41 \cdot 10^{-3}$ & 2.09 & $8.04 \cdot 10^{-4}$ \\
9 & 0.152 & $8.54 \cdot 10^{-3}$ & 1.64 & $7.87 \cdot 10^{-3}$ \\
10 & 0.159 & $2.5 \cdot 10^{-4}$ & 0.73 & $2.51 \cdot 10^{-4}$ \\
11 & 0.162 & $8.41 \cdot 10^{-3}$ & 1.50 & $8.69 \cdot 10^{-3}$ \\
12 & 0.176 & $9.30 \cdot 10^{-3}$ & 1.43 & $1.14 \cdot 10^{-2}$ \\
13 & 0.185 & $9.00 \cdot 10^{-3}$ & 1.38 & $1.22 \cdot 10^{-2}$ \\
14 & 0.268 & $3.80 \cdot 10^{-3}$ & 1.80 & $1.08 \cdot 10^{-2}$ \\
15 & 0.306 & $2.20 \cdot 10^{-3}$ & 3.72 & $8.18 \cdot 10^{-3}$ \\
16 & 0.322 & $2.10 \cdot 10^{-3}$ & 1.14 & $8.64 \cdot 10^{-3}$ \\
\hline
\end{tabular}

Table 5. Lowest sixteen nondimensional natural frequencies, modal masses, damping ratios, and stiffnesses of the system including linear visco-elastic vibration absorbers optimized according to the nonsymmetric point-load distribution.

\begin{tabular}{c|cccc} 
Mode & $\bar{f}[-]$ & $\bar{M}[-]$ & $\zeta[\%]$ & $\bar{K}[-]$ \\
\hline 1 & 0.057 & $1.59 \cdot 10^{-3}$ & 1.84 & $2.09 \cdot 10^{-4}$ \\
2 & 0.061 & $1.14 \cdot 10^{-3}$ & 2.05 & $1.69 \cdot 10^{-4}$ \\
3 & 0.090 & $3.31 \cdot 10^{-3}$ & 1.89 & $1.07 \cdot 10^{-3}$ \\
4 & 0.097 & $6.20 \cdot 10^{-4}$ & 3.15 & $2.30 \cdot 10^{-4}$ \\
5 & 0.112 & $1.29 \cdot 10^{-3}$ & 2.08 & $6.48 \cdot 10^{-4}$ \\
6 & 0.120 & $1.37 \cdot 10^{-3}$ & 2.09 & $7.79 \cdot 10^{-4}$ \\
7 & 0.152 & $8.55 \cdot 10^{-3}$ & 1.64 & $7.88 \cdot 10^{-3}$ \\
8 & 0.159 & $2.50 \cdot 10^{-4}$ & 0.72 & $2.54 \cdot 10^{-4}$ \\
9 & 0.162 & $8.19 \cdot 10^{-3}$ & 1.50 & $8.53 \cdot 10^{-3}$ \\
10 & 0.176 & $8.89 \cdot 10^{-3}$ & 1.41 & $1.09 \cdot 10^{-2}$ \\
11 & 0.178 & $6.00 \cdot 10^{-4}$ & 0.81 & $7.46 \cdot 10^{-4}$ \\
12 & 0.185 & $8.46 \cdot 10^{-3}$ & 1.37 & $1.15 \cdot 10^{-2}$ \\
13 & 0.263 & $3.43 \cdot 10^{-3}$ & 2.93 & $9.39 \cdot 10^{-3}$ \\
14 & 0.296 & $2.18 \cdot 10^{-3}$ & 3.91 & $7.58 \cdot 10^{-3}$ \\
15 & 0.318 & $1.05 \cdot 10^{-3}$ & 0.80 & $4.20 \cdot 10^{-3}$ \\
16 & 0.327 & $6.80 \cdot 10^{-4}$ & 1.22 & $2.87 \cdot 10^{-3}$ \\
\hline
\end{tabular}

of points $A_{j}, B_{k}$ and $C$ are considered in the minimization of the objective function in Eq. (9), while those induced in the roller $P_{i}$ are neglected since they cannot be directly controlled by ad hoc VAs positioned atop each of these points due to physical constraints.

The solutions obtained for the linearly visco-elastic VAs (linearized system) are used to set the search space for the parameters of the nonlinearly visco-elastic absorbers. The damping coefficient and the stiffness of the nonlinear absorbers (see Eqs. (6) and (7)) can be expressed as a function of the linearized frequencies $\bar{f}_{n}$, damping ratios $\zeta_{n}$, and masses $m_{n}$ as follows: $d_{n}=4 \pi \zeta_{n} \bar{f}_{n} m_{n}$ and $k_{n}=$ $\left(2 \pi \bar{f}_{n}\right)^{2} m_{n}$. In this way, the optimization of the nonlinear
VAs can be compared with the results obtained for the linear VAs according to the harmonic solutions. It is worth recalling, that the linearization of the nonlinear VAs restoring forces provides those of the linear absorbers. Moreover, when the parameters $\xi_{k, n}$ and $\xi_{d, n}$ are equal to zero, the nonlinear absorbers exhibit the same restoring forces of the linear case.

The DE-based optimization of the parameters $\bar{f}_{n}, \zeta_{n}$, $\xi_{k, n}, \xi_{d, n}$, and $x_{n}$ is performed by assuming 25 values for each search space and setting the VAs masses to the values reported in Tab. 2. Table 6 shows the optimal parameters found for the nonlinearly visco-elastic absorbers considering the vehicle running at the nondimensional speed of 0.025 . The objective function $\Gamma$ converges to the minimum after 500 iterations (see Fig. 15). Figures 16, 17 and 18 show the time histories of the accelerations of $P_{1}, P_{3}, A_{2}, A_{3}, B_{1}$ and $C$ for the uncontrolled and the controlled cases, respectively. The force-displacement cycles of the nonlinear VAs positioned at $A_{j}, B_{k}$ and $C$ are reported in Figs. 19, 20, and 21.

According to the optimal values of $\xi_{k, n}$ reported in Tab. 6 , the VA positioned at $A_{1}$ exhibits a limited hardening behavior while the remaining VAs show a limited softening restoring force. In particular, the absorber at $A_{4}$ exhibits a linear visco-elastic behavior because of the small value of $x_{n}$ that provides a fast convergence to a constant stiffness and damping. A linear visco-elastic behavior can be observed also for the absorbers at $B_{2}$ and $C$. This is due to the small displacements range characterizing their oscillations. The coefficient $\xi_{d, n}$ is greater than zero for all absorbers except for that at $B_{2}$. Thus, the optimization procedure drives the damping behavior of the VAs so as to reduce the damped energy in the small displacements range.

The control provided by the set of nonlinearly viscoelastic VAs is effective. The analysis of Tabs. 7 and 8 show that the peak accelerations and the mean accelerations are consistently reduced with respect to the uncontrolled case. The linearly visco-elastic absorbers optimized for the harmonic nonsymmetric loading distribution and tested in the nonlinear ropeway system with the vehicle transit show lower performance if compared to that of the nonlinearly visco-elastic VAs. The only point of the system for which the maximum acceleration is amplified for both linear and nonlinear VAs is $B_{2}$.

The obtained results can be explained as follows. The absorber at $A_{1}$ is the first affected by the impact of the grip. The mild hardening with low stiffness and damping for small displacements facilitates the energy transfer from the roller to the absorber. The other absorbers, including that at $A_{2}$, which are excited for the first time moderately by the initial impact, respond showing a mild softening dynamic behavior, in accordance with the softening nonlinearities of the roller battery system due to the inertia nonlinear effects [23].

\section{CONCLUSIONS}

A multi-body dynamic model of a compression rollerbattery that is part of a mono-cable ropeway was employed to investigate a vibration control strategy making use of multiple linearly and nonlinearly visco-elastic VAs. The stiffness and viscosity parameters of the linear VAs were designed by means of a Differential Evolution 
Table 6. Optimized parameters of the nonlinearly visco-elastic VAs for the vehicle transit across the roller at the velocity of 0.025

\begin{tabular}{cccccc} 
VA & $\bar{f}_{\text {opt }}$ & $\zeta_{\text {opt }}$ & $\xi_{k, \text { opt }}$ & $\xi_{d, o p t}$ & $x_{\text {opt }}$ \\
& {$[-]$} & {$[\%]$} & {$[-]$} & {$[-]$} & {$[-]$} \\
\hline$A_{1}$ & 0.123 & 3.43 & 0.87 & 0.94 & 0.0250 \\
$A_{2}$ & 0.120 & 2.15 & -0.35 & 0.78 & 0.0136 \\
$A_{3}$ & 0.114 & 1.95 & -0.64 & 0.13 & 0.0096 \\
$A_{4}$ & 0.118 & 1.46 & -0.29 & 0.95 & 0.0004 \\
$B_{1}$ & 0.091 & 0.51 & -0.95 & 0.95 & 0.0362 \\
$B_{2}$ & 0.186 & 0.50 & -0.75 & -0.79 & 0.0342 \\
$C$ & 0.281 & 0.78 & -0.95 & 0.31 & 0.0121 \\
\hline
\end{tabular}

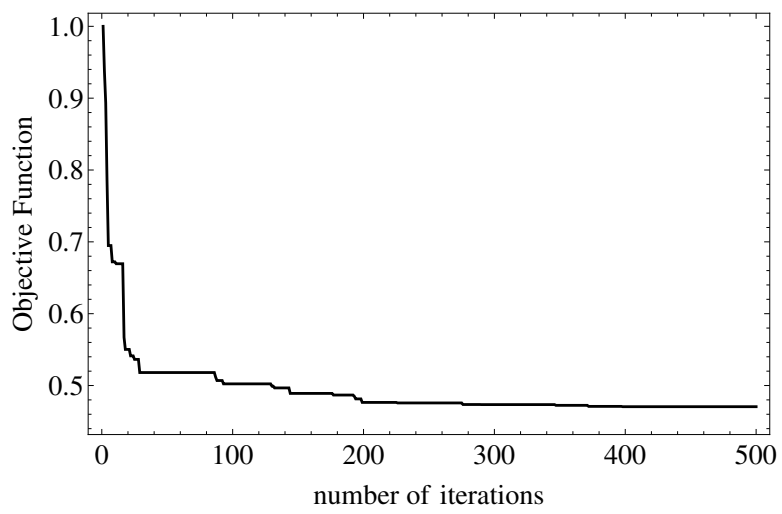

Figure 15. Normalized objective function vs. number of iterations performed by the DE algorithm for the optimization of the nonlinearly visco-elastic absorbers.
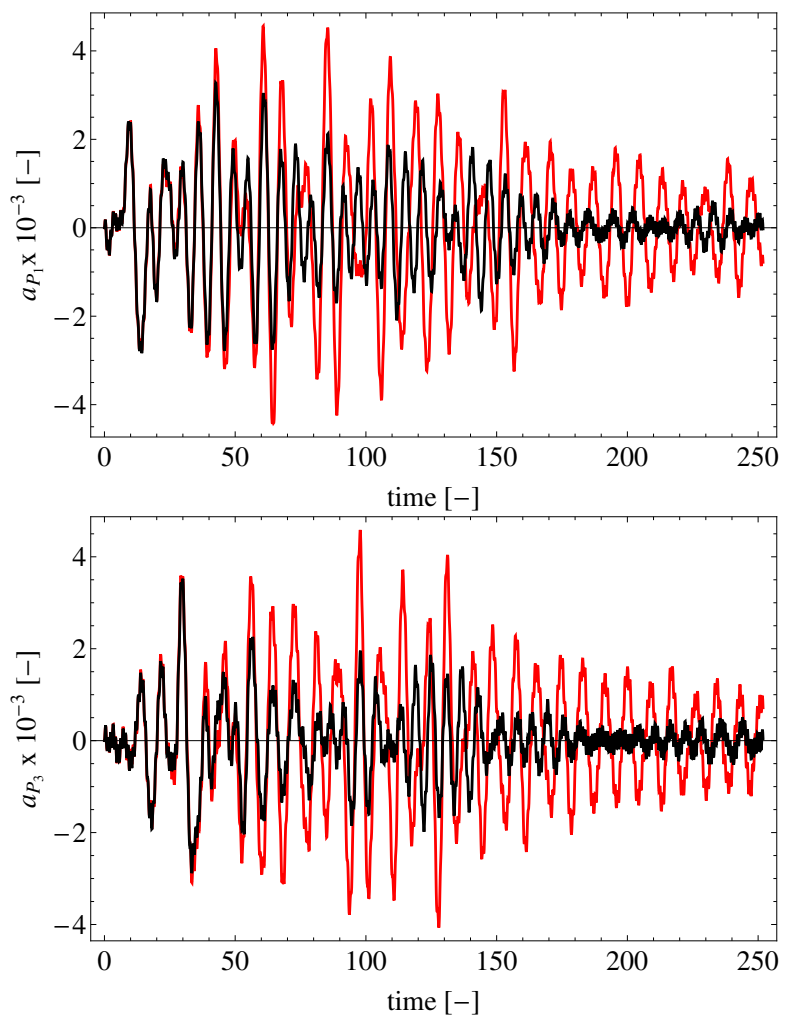

Figure 16. Acceleration vs. time at $P_{1}$ (top) and $P_{3}$ (bottom) for the vehicle speed of 0.025 : in the uncontrolled (red line) and controlled (black line) via nonlinearly visco-elastic VAs.
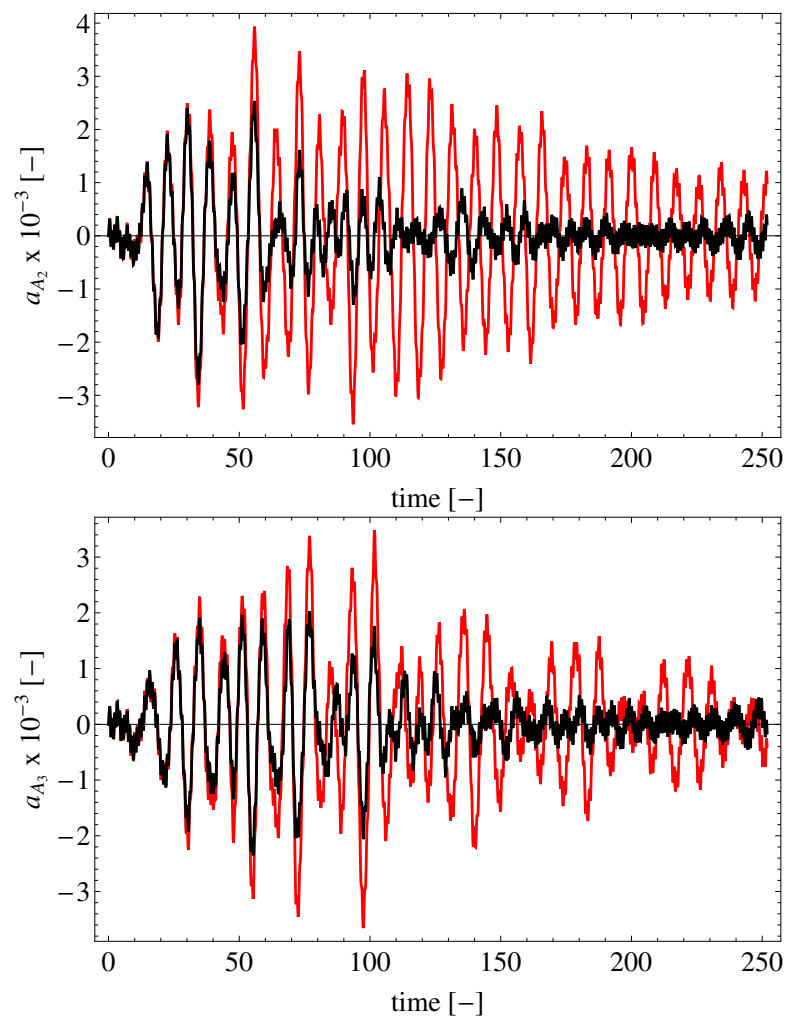

Figure 17. Acceleration vs. time at $A_{2}$ (top) and $A_{3}$ (bottom) for the vehicle speed of 0.025: in the uncontrolled (red line) and controlled (black line) via nonlinearly visco-elastic VAs.
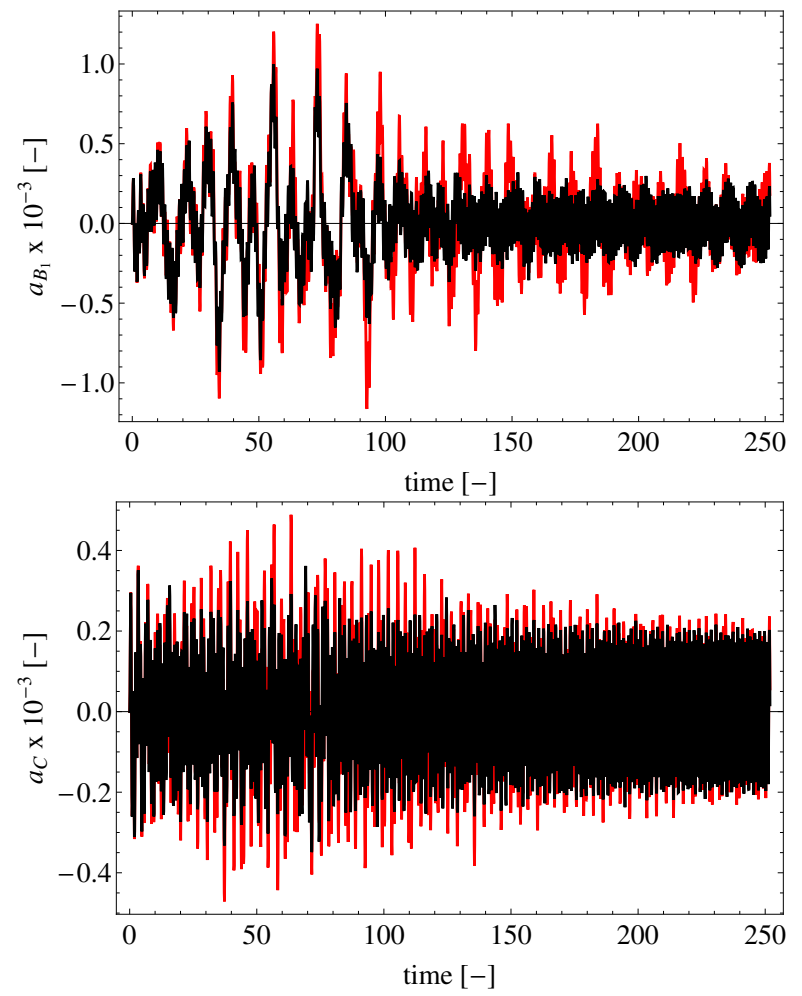

Figure 18. Acceleration vs. time at $B_{1}$ (top) and $C$ (bottom) for the vehicle speed of 0.025 : in the uncontrolled (red line) and controlled (black line) via nonlinearly visco-elastic VAs.

optimization seeking to minimize the linear frequency response functions for the accelerations of the control points under harmonic excitations. A novel mechanical 

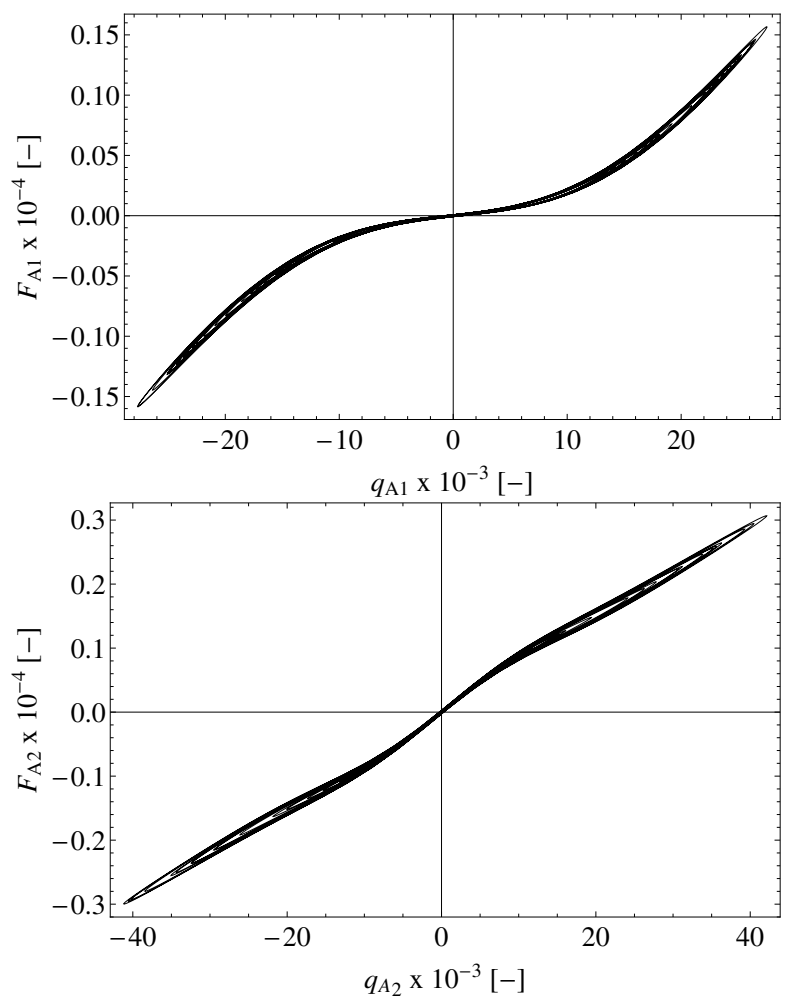

Figure 19. Force-displacement cycles for nonlinearly visco-elastic VAs at $A_{1}$ (top) and $A_{2}$ (bottom) for the vehicle speed of 0.025 .
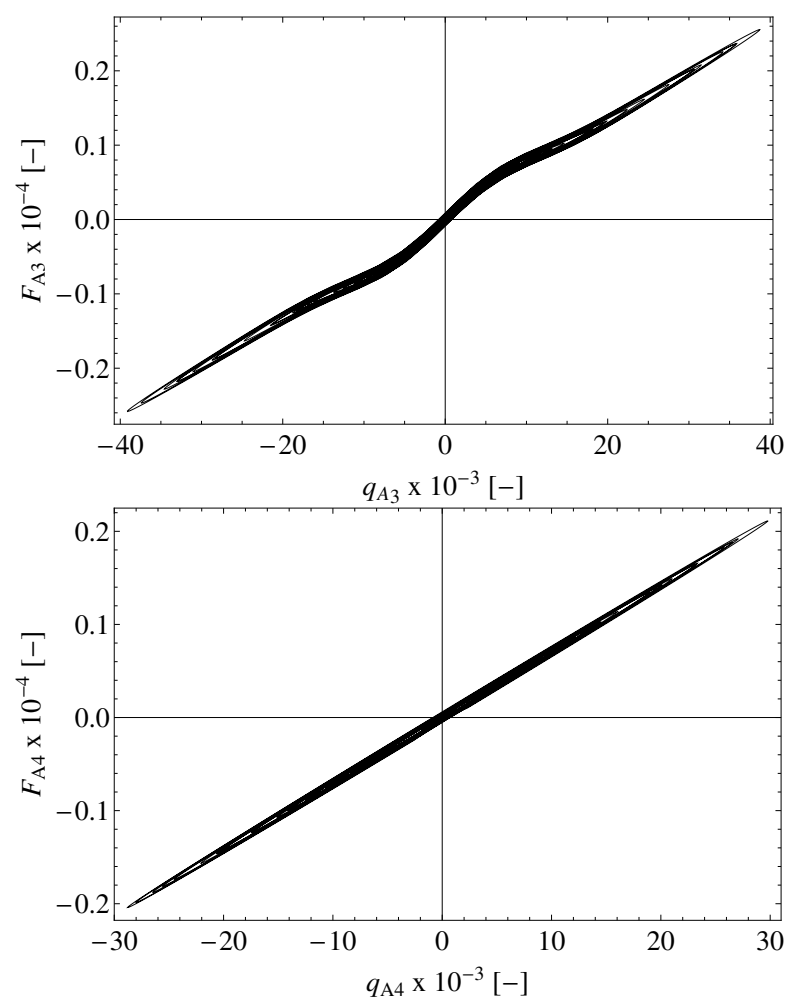

Figure 20. Force-displacement cycles for nonlinearly visco-elastic VAs at $A_{3}$ (top) and $A_{4}$ (bottom) for the vehicle speed of 0.025 .

model of vibration absorber exhibiting a nonlinearly viscoelastic (hysteretic-like) behavior is proposed. The innovative device can provide a mild hardening or softening behavior combined with a modulation of the energy dissipation
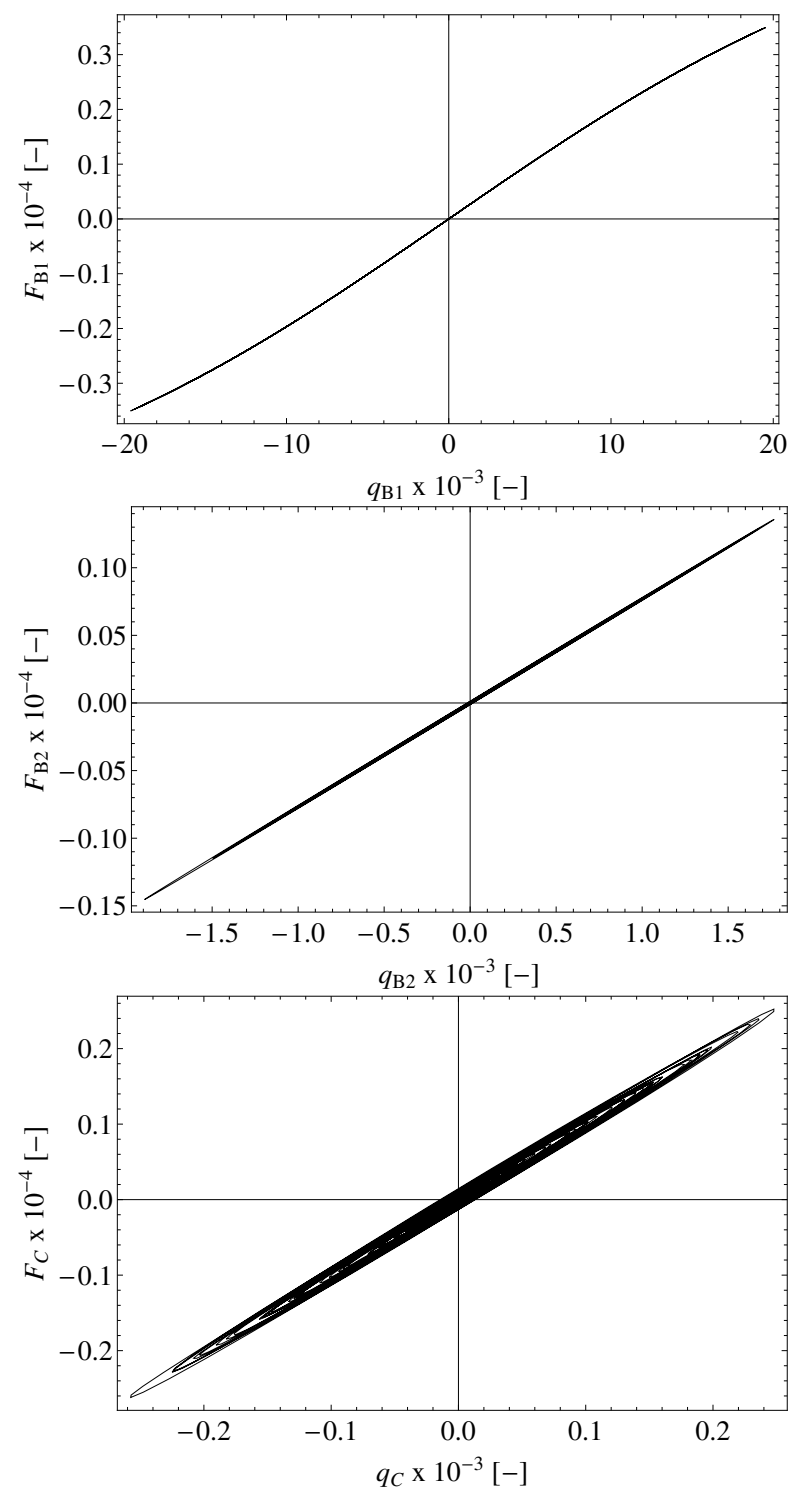

Figure 21. Force-displacement cycles for nonlinearly visco-elastic VAs at $B_{1}$ (top), $B_{2}$ (middle) and $C$ (bottom) for the vehicle speed of 0.025 .

rate across the force-displacement cycles. The parameters of the nonlinearly visco-elastic absorbers were estimated performing a Differential Evolution optimization on the fully nonlinear system for the passage of the vehicle across the roller at a relatively high speed. Finally, the performances of the linear VAs, optimized through the linear FRCs for the nonsymmetric loading condition, are compared with the nonlinear VAs in controlling the nonlinear system excited by the transit of the vehicle.

The results showed that the proposed nonlinear absorbers are highly efficient in the mitigation of the accelerations induced by the vehicle transit in the roller mechanical parts and perform better than linear optimal devices. An important aspect to highlight is represented by the results of the optimization of the nonlinear VAs. The combination of mild softening and hardening with low dissipation rates in a small oscillations range seems to be a good way forward for controlling both the transient and the stationary dynamic response of the nonlinear mechanical system. The mild lowstiffness hardening behavior is suitable for the mitigation of 
Table 7. Comparison between the mean accelerations (i.e., Mean(a)) calculated in the uncontrolled case and in the case controlled by linear and nonlinear visco-elastic VAs, respectively, for the vehicle nondimensional transit speed of 0.025 .

\begin{tabular}{cccc}
\hline & Uncontrolled & Nonlinear VAs & Linear VAs \\
\hline Point & Mean $(\mathrm{a})[-]$ & Mean $(\mathrm{a})[-]$ & Mean $(\mathrm{a})[-]$ \\
\hline$P_{1}$ & $1.28 \cdot 10^{-3}$ & $7.06 \cdot 10^{-4}$ & $8.43 \cdot 10^{-4}$ \\
$P_{2}$ & $1.08 \cdot 10^{-3}$ & $9.38 \cdot 10^{-4}$ & $9.35 \cdot 10^{-4}$ \\
$P_{3}$ & $1.20 \cdot 10^{-3}$ & $5.45 \cdot 10^{-4}$ & $7.44 \cdot 10^{-4}$ \\
$P_{4}$ & $1.36 \cdot 10^{-3}$ & $7.13 \cdot 10^{-4}$ & $9.11 \cdot 10^{-4}$ \\
$P_{5}$ & $8.82 \cdot 10^{-4}$ & $6.63 \cdot 10^{-4}$ & $7.18 \cdot 10^{-4}$ \\
$P_{6}$ & $1.27 \cdot 10^{-3}$ & $7.20 \cdot 10^{-4}$ & $9.08 \cdot 10^{-4}$ \\
$P_{7}$ & $7.12 \cdot 10^{-4}$ & $7.23 \cdot 10^{-4}$ & $7.05 \cdot 10^{-4}$ \\
$P_{8}$ & $1.09 \cdot 10^{-3}$ & $7.24 \cdot 10^{-4}$ & $8.72 \cdot 10^{-4}$ \\
$A_{1}$ & $9.06 \cdot 10^{-4}$ & $3.37 \cdot 10^{-4}$ & $5.31 \cdot 10^{-4}$ \\
$A_{2}$ & $1.19 \cdot 10^{-3}$ & $4.09 \cdot 10^{-4}$ & $6.93 \cdot 10^{-4}$ \\
$A_{3}$ & $8.77 \cdot 10^{-4}$ & $4.44 \cdot 10^{-4}$ & $5.87 \cdot 10^{-4}$ \\
$A_{4}$ & $5.84 \cdot 10^{-4}$ & $3.03 \cdot 10^{-4}$ & $3.95 \cdot 10^{-4}$ \\
$B_{1}$ & $2.55 \cdot 10^{-4}$ & $1.83 \cdot 10^{-4}$ & $2.05 \cdot 10^{-4}$ \\
$B_{2}$ & $2.51 \cdot 10^{-4}$ & $1.86 \cdot 10^{-4}$ & $2.05 \cdot 10^{-4}$ \\
$C$ & $1.39 \cdot 10^{-4}$ & $1.26 \cdot 10^{-4}$ & $1.26 \cdot 10^{-4}$ \\
\hline
\end{tabular}

Table 8. Comparison between the peak accelerations (i.e., Max(a)) calculated in the uncontrolled case and in the case controlled by linear and nonlinear visco-elastic VAs, respectively, for the vehicle nondimensional transit speed of 0.025 .

\begin{tabular}{cccc}
\hline & Uncontrolled & Nonlinear VAs & Linear VAs \\
\hline Point & $\operatorname{Max}(\mathrm{a})[-]$ & $\operatorname{Max}(\mathrm{a})[-]$ & $\operatorname{Max}(\mathrm{a})[-]$ \\
\hline$P_{1}$ & $4.55 \cdot 10^{-3}$ & $3.28 \cdot 10^{-3}$ & $3.76 \cdot 10^{-3}$ \\
$P_{2}$ & $4.97 \cdot 10^{-3}$ & $5.05 \cdot 10^{-3}$ & $5.07 \cdot 10^{-3}$ \\
$P_{3}$ & $4.58 \cdot 10^{-3}$ & $3.52 \cdot 10^{-3}$ & $3.61 \cdot 10^{-3}$ \\
$P_{4}$ & $5.72 \cdot 10^{-3}$ & $4.65 \cdot 10^{-3}$ & $5.24 \cdot 10^{-3}$ \\
$P_{5}$ & $5.10 \cdot 10^{-3}$ & $4.31 \cdot 10^{-3}$ & $4.59 \cdot 10^{-3}$ \\
$P_{6}$ & $6.00 \cdot 10^{-3}$ & $4.53 \cdot 10^{-3}$ & $5.36 \cdot 10^{-3}$ \\
$P_{7}$ & $5.74 \cdot 10^{-3}$ & $4.82 \cdot 10^{-3}$ & $5.33 \cdot 10^{-3}$ \\
$P_{8}$ & $8.90 \cdot 10^{-3}$ & $6.72 \cdot 10^{-3}$ & $7.79 \cdot 10^{-3}$ \\
$A_{1}$ & $3.07 \cdot 10^{-3}$ & $1.90 \cdot 10^{-3}$ & $2.45 \cdot 10^{-3}$ \\
$A_{2}$ & $3.93 \cdot 10^{-3}$ & $2.80 \cdot 10^{-3}$ & $3.31 \cdot 10^{-3}$ \\
$A_{3}$ & $3.65 \cdot 10^{-3}$ & $2.35 \cdot 10^{-3}$ & $2.91 \cdot 10^{-3}$ \\
$A_{4}$ & $2.74 \cdot 10^{-3}$ & $1.66 \cdot 10^{-3}$ & $2.13 \cdot 10^{-3}$ \\
$B_{1}$ & $1.25 \cdot 10^{-3}$ & $1.89 \cdot 10^{-3}$ & $2.45 \cdot 10^{-3}$ \\
$B_{2}$ & $1.06 \cdot 10^{-3}$ & $2.80 \cdot 10^{-3}$ & $3.31 \cdot 10^{-3}$ \\
$C$ & $4.88 \cdot 10^{-4}$ & $3.61 \cdot 10^{-4}$ & $3.56 \cdot 10^{-4}$ \\
\hline
\end{tabular}

the transient response, while the limited softening, which tailors the nonlinearities of the roller battery system, controls better the stationary dynamics.

The obtained results show the feasibility of a multimode vibration control strategy for a ropeway transportation system. The achieved vibration mitigation can have a significant impact on the maintenance operations and associated costs due to the increased dynamical resilience of the mechanical parts. Moreover, it is possible to operate the ropeway with higher running speeds thus increasing the throughput of the system. The proposed prototype of absorber can be actually realized and installed on a ropeway at relatively low cost.

\section{Acknowledgements}

This work was partially supported by the Italian Ministry of Education, University and Scientific Research. under PRIN Grant No. 2017L7X3CS. Dr. Andrea Arena gratefully acknowledges partial support through the Assistant Professor Sapienza Fellowship program under contract "Nonlinear dynamics of cable network systems". The project "Dynamics of towers - Feasibility of vibration absorbers" supported by POMA (Dr. Mathieu Babaz) is gratefully acknowledged.

\section{References}

[1] Alshalalfah BW, Shalaby AS, Dale S et al. Feasibility study of aerial ropeway transit in the holy city of makkah. Transportation Planning and Technology 2015; 38(4): 392-408.

[2] Hoffmann K and Liehl R. Cable-drawn urban transport systems. WIT Transactions on The Built Environment 2005; 77: 25-36.

[3] Hoffmann K. Recent developments in cable-drawn urban transport systems. FME Transactions 2006; 34(4): 205-212.

[4] Hoffmann K, Gabmayer T and Huber G. Measurement of oscillation effects on ropeways and chairlifts. ÖIAZ 2008; 153(10-12): 435-438.

[5] Hoffmann K. Oscillation effects of ropeways caused by cross-wind and other influences. FME Transactions 2009; 37: 175-184.

[6] Bryja D and Knawa M. Computational model of an inclined aerial ropeway and numerical method for analyzing nonlinear cable-car interaction. Computers and Structures 2011; 89: 1895-1905.

[7] Nan C, Meyer-Piening HR and Decking C. Dynamic behaviour of cable supporting roller batteries: Basic model. Computers and Structures 1998; 69: 95-104.

[8] Arena A, Casalotti A, Lacarbonara W et al. Dynamics of container cranes: Three-dimensional modeling, fullscale experiments, and identification. International Journal of Mechanical Sciences 2015; 93: 8-21.

[9] Arena A, Lacarbonara W and Cartmell M. Nonlinear interactions in deformable container cranes. Proc IMechE Part C: Journal of Mechanical Engineering Science 2016; 230(1): 5-20.

[10] Arena A, Lacarbonara W and Casalotti A. Payload oscillations control in harbor cranes via semiactive vibration absorbers: Modeling, simulations and experimental results. Procedia Engineering 2017; 199: 501-509.

[11] Arena A, Carboni B, Lacarbonara W et al. Dynamic response and identification of tower-cable-roller battery interactions in ropeways. In Proceedings of the ASME Design Engineering Technical Conference, IDETC/CIE 2017, volume 6. ASME. 
[12] Arena A, Carboni B, Angeletti F et al. Ropeway roller batteries dynamics: Modeling, identification, and fullscale validation. Engineering Structures 2019; 180: 793-808.

[13] Storn R. On the usage of differential evolution for function optimization. In Proceedings of the Biennial Conference of the North American Fuzzy Information Processing Society. pp. 519-523.

[14] Storn R and Price K. Differential evolution a simple and efficient heuristic for global optimization over continuous spaces. Journal of Global Optimization 1997; 11(4): 341-359.

[15] Carboni B and Lacarbonara W. Nonlinear vibration absorber with pinched hysteresis: Theory and experiments. Journal of Engineering Mechanics 2016; 142(5): 04016023. DOI:10.1061/(ASCE)EM.19437889.0001072 .

[16] Carboni B and Lacarbonara W. Nonlinear dynamic characterization of a new hysteretic device: experiments and computations. Nonlinear Dynamics 2016; 83(1-2): 23-39.

[17] Carboni B, Arena A and Lacarbonara W. Passive vibration control of roller batteries in cableways. In ASME 2018 International Design Engineering Technical Conferences and Computers and Information in Engineering Conference. American Society of Mechanical Engineers Digital Collection.

[18] Lacarbonara W and Carboni B. Multi-performance hysteretic rheological device. Technical report, Sapienza pending patent n. EP3259489B1US20180245655A1-CN107864662A, 2019.

[19] Den Hartog JP. Mechanical vibrations. Courier Corporation, 1985.

[20] Ioi T and Ikeda K. On the dynamic vibration damped absorber of the vibration system. Bulletin of JSME 1978; 21(151): 64-71.

[21] Carboni B, Lacarbonara W and Auricchio F. Hysteresis of multiconfiguration assemblies of nitinol and steel strands: Experiments and phenomenological identification. Journal of Engineering Mechanics 2015; 141(3).

[22] Bagheri S and Rahmani-Dabbagh V. Seismic response control with inelastic tuned mass dampers. Engineering Structures 2018; 172: 712-722.

[23] Arena A and Lacarbonara W. Nonlinear models of roller batteries and experimental validation. In Symposium "Nonlinear Dynamics-Scientific work of Prof. Dr Katica (Stevanovic) Hedrih" Mathematical Institute of SASA, Belgrade, 04.-06. September, 2019. 